\title{
SINGLE PHASE ENERGY MINIMIZERS FOR MATERIALS WITH NONLOCAL SPATIAL DEPENDENCE
}

\author{
By \\ ROGER L. FOSDICK AND DARREN E. MASON
}

Department of Aerospace Engineering and Mechanics, University of Minnesota, Minneapolis, MN 55455

\begin{abstract}
We consider a one-dimensional body and assume that the total stored energy functional depends not only on the local strain field but also on the spatial average of the strain field over the body, weighted with an influence kernel. We investigate the problem of minimizing the total stored energy subject to given end displacements. The general existence theory for this problem is reviewed. Then, we narrow our study and concentrate on certain fundamental aspects of nonlocal spatial dependence by restricting our consideration to the case of a convex local energy and an exponential-type influence function for the nonlocal part. We find explicit solutions and show their characteristic properties as a function of the parameter that measures the extent of influence in the nonlocal kernel. We then study in detail the behavior that results when the total stored energy functional loses its coercivity. In this case, issues concerning the local and global stability of extremal fields are considered.
\end{abstract}

Table of contents

1. Introduction

2. The reduced model: A necessary differential equation

3. The structure of admissible extremals

4. Analysis of minimizers in the Cases (1) and (2): $k \geq 2 a$

5. The minimization problem in the Case (3): $k<2 a$

5.1. General extremal fields: Existence and asymptotics

5.2. Stability analysis

1. Introduction. Consider a one-dimensional body $\mathscr{B}$ represented in its reference configuration by the point set

$$
\mathscr{B}:=(0, l) \subset \mathbb{R},
$$

where $\mathbb{R}$ represents the set of all real numbers. Let $x \in \mathscr{B}$ denote a material point of $\mathscr{B}$ and let a deformation of $\mathscr{B}$ be given by a continuous mapping $y(\cdot) \in \mathscr{C}(\mathscr{B}, \mathbb{R})$. 
Then, $u(x):=y(x)-x$ is the associated displacement field for all $x \in \mathscr{B}$ and we let $e(\cdot):=u^{\prime}(\cdot)$ denote the strain field. A common hypothesis in the theory of materials is to assume that $\mathscr{B}$ supports a certain total stored energy functional $E(\cdot): \mathscr{D} \longrightarrow$ $\mathbb{R} \cup\{\infty\}$ where $\mathscr{D}$ is a given class of continuous displacement fields in $\mathscr{C}(\mathscr{B}, \mathbb{R})$. In such theories, the stable equilibrium states of the body $\mathscr{B}$ are then supposed to be those displacement fields that minimize $E(\cdot)$ over $\mathscr{D}$ subject to given boundary conditions. In this work we are interested in the fixed displacement problem, for which $\Delta \in \mathbb{R}^{+}:=\{x \in \mathbb{R}: x \geq 0\}$ is given, and we seek a displacement field $u_{0}(\cdot) \in \mathscr{A}_{>}(\mathscr{B})$ such that

$$
E\left(u_{0}\right)=\min _{u \in \mathscr{A}_{>}(\mathscr{B})} E(u)
$$

where

$$
\mathscr{A}_{>}(\mathscr{B}):=\left\{u(\cdot) \in \mathscr{D}: \int_{\mathscr{B}} e(x) d x=\Delta \text { and } e(x)>-1 \text { for a.e. } x \in \mathscr{B}\right\} .
$$

Here, we require $e(x)>-1$ to ensure the invertibility of the deformation $y(\cdot)$. The particular functional form of the total stored energy functional $E(\cdot)$ will invariably affect the class of displacements $\mathscr{D}$ over which the minimum is sought.

A variety of forms for the total stored energy functional $E(\cdot)$ have been considered in recent years. In $[6,8]^{1}$, the body $\mathscr{B}$ is assumed to be elastic and the total stored energy functional consists of the integral over $\mathscr{B}$ of a sole nonconvex integrand composed with the strain field $e(\cdot)$. In that case, the set $\mathscr{D}$ may be taken to be the Sobolev space $W^{1, p}(\mathscr{B}), 1<p<\infty$, and the problem (1) generally leads to highly nonunique minimizers. In fact, for certain $\Delta>0$ there are minimizers that oscillate wildly between two different values of strain on subsets of the body $\mathscr{B}$ that are fixed only in measure. A method of removing such indeterminateness is to modify the total stored energy functional so as to include a penalty regularization that participates in the minimization and, in a sense, forms the basis of a selection criterion. This approach has been employed in two different, but related, attempts by Gurtin, Slemrod, and Carr [3] and by Walter [13]. In the first, higher-order gradients are introduced into the total stored energy functional, thereby creating a penalty (through increased energy) for the formation of regions where rapid changes in the strain field occur. In the second approach, the dependence of the total stored energy on a localized gradient is generalized and replaced by dependence on the spatial average of the strain field weighted with an influence kernel. This approach requires less hypotheses on the local smoothness of admissible strain fields and, in a firstorder approximation for smooth fields, is arguably reducible to the gradient model. We shall adopt the nonlocal approach here.

Specifically, we suppose that the total stored energy functional for $\mathscr{B}$ is of the form

$$
E_{\delta}(u):=\int_{\mathscr{B}}\left\{W(e(x))-e(x) \int_{\mathscr{B}} f_{\delta}(x-z) e(z) d z\right\} d x,
$$

\footnotetext{
'The work of [6] concerns the question of coexistent phases in Gibbsian thermostatics and thermodynamics and is more general than implied here. In particular, it addresses the elementary thermoelastic bar problem for nonconvex energies.
} 


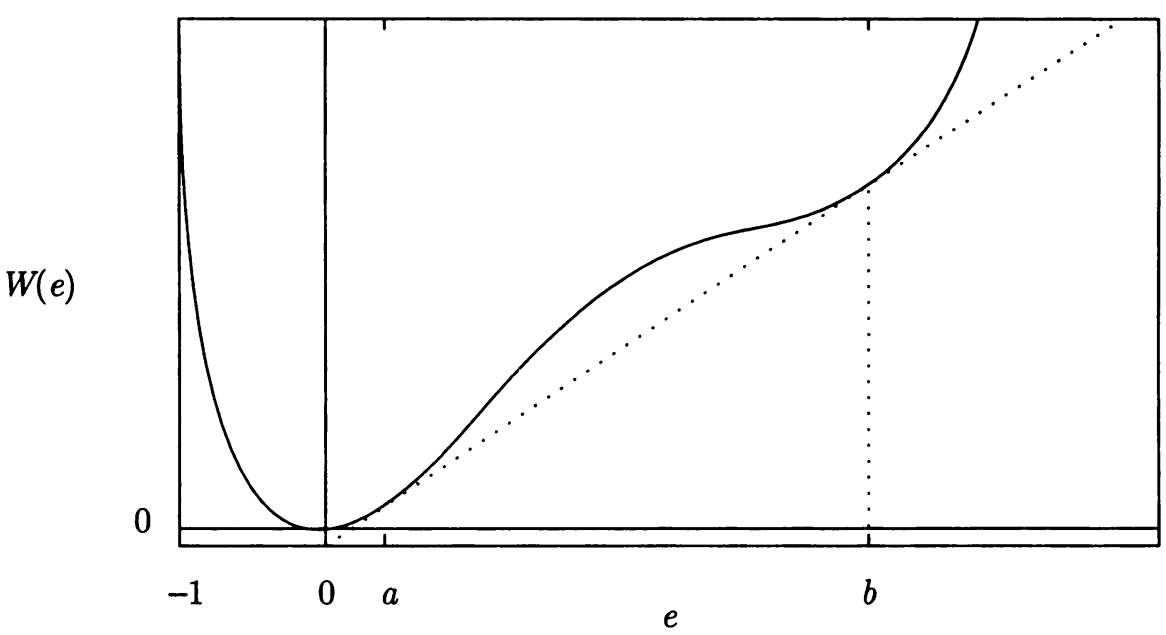

FIG. 1a. The bulk stored energy $W(e)$

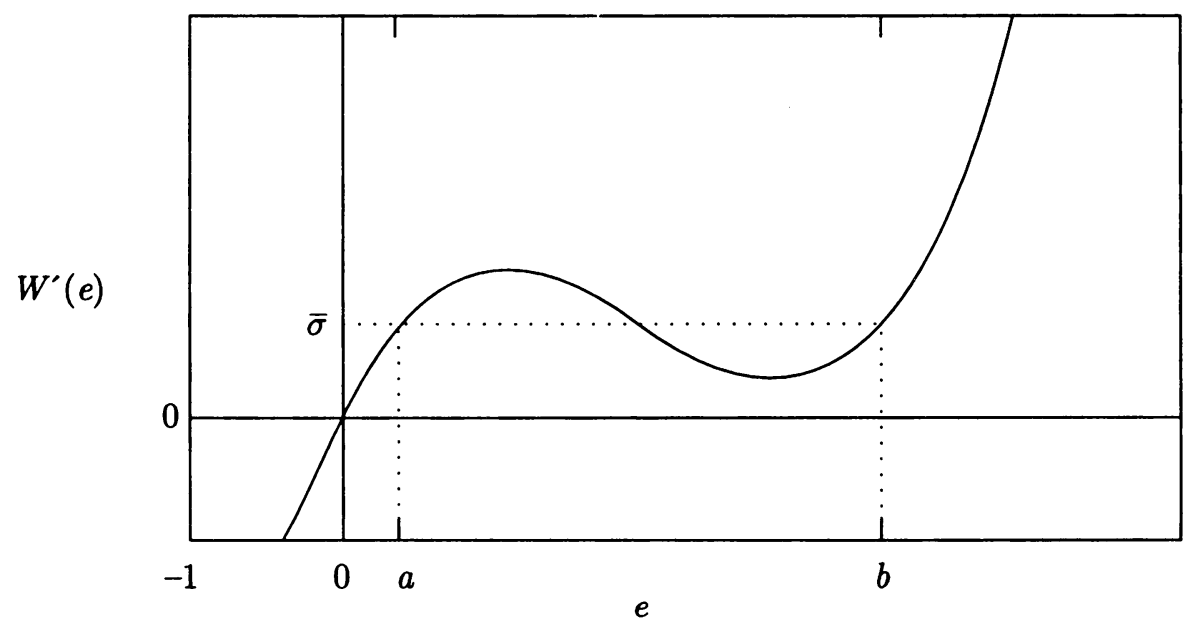

FIG. 1b. The derivative $W^{\prime}(e)$ of the bulk stored energy $W(e)$

where $e(x):=u^{\prime}(x)$ for every $x \in \mathscr{B}$. Here, $W(\cdot)$ represents the local pointwise contribution of the strain field to the total stored energy of $\mathscr{B}$. This portion of $E_{\delta}(\cdot)$ usually is referred to as the bulk or local energy (Figs. 1a and $1 \mathrm{~b}$ ). In general, $W(\cdot): \mathbb{R} \rightarrow \mathbb{R}^{+} \cup\{\infty\}$ is assumed to satisfy

Hypothesis 1 (BUlK ENERGY). $W(\cdot): \mathbb{R} \rightarrow \mathbb{R}^{+} \cup\{\infty\}$ is a continuously differentiable function such that

(i)

$$
\lim _{e \rightarrow \infty} W(e)=\lim _{e \rightarrow \infty} W^{\prime}(e)=\infty
$$

(ii) $\exists: a, b \in(0, \infty)$ such that $0<a<b<\infty$ and $W(\cdot)$ is convex on $(-\infty, a$ ] $\cup[b, \infty)$.

The second term in (3) represents the nonlocal part of the constitutive assumption and expresses the spatial strain dependence via an influence function $f_{\delta}(\cdot)$. Given 


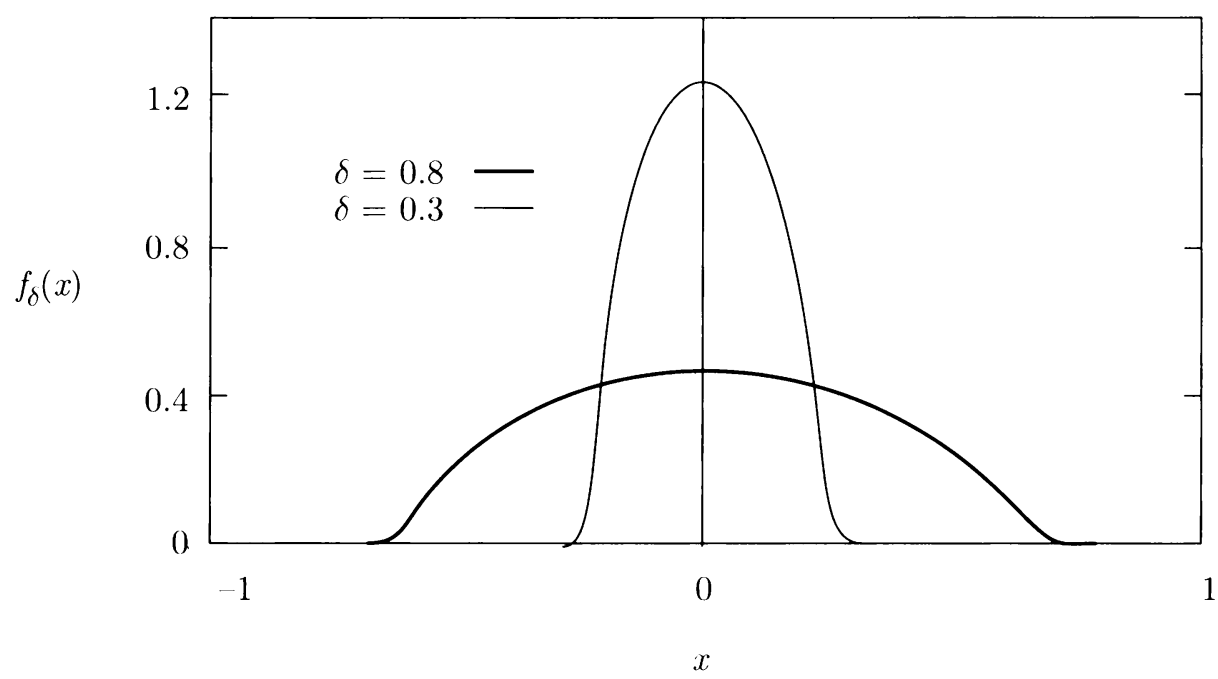

FIG. 2. A possible influence function: $f_{\delta}(x):=\frac{1}{\delta} e^{\left\{\left((x / \delta)^{2}-1\right)^{-1}\right\}}$ for $|x|<\delta=0.3,0.8$.

$x \in \mathscr{B}$, the function $f_{\delta}(x-z)$ in (3) serves as a weight on the relative influence of the material strain $e(z)$ at a point $z$ in a $\delta$-neighborhood of $x$. Specifically, $f_{\delta}(\cdot)$ behaves as a weighted identity approximation and is assumed to satisfy

Hypothesis 2 (Spatial Energy). For $2 \leq p<\infty, f_{\delta}(\cdot) \in L^{q}(\mathbb{R})$ is a nonnegative, monotonically decreasing even function with the following properties:

(i) $\exists \bar{a}>0$ such that $\int_{\mathbb{R}} f_{\delta}(x) d x=\bar{a}, \quad \forall \delta>0$ (fixed total spatial influence),

(ii) $\forall \phi(\cdot) \in L^{p}(\mathscr{B}), \int_{\mathscr{B}} f_{\delta}(x-y) \phi(y) d y \rightarrow 0$ in $L^{q}(\mathscr{B})$ as $\delta \rightarrow \infty$,

(iii) $\forall \phi(\cdot) \in L^{p}(\mathscr{B}), \int_{\mathscr{B}} f_{\delta}(x-y) \phi(y) d y \rightarrow \bar{a} \phi(x)$ in $L^{q}(\mathscr{B})$ as $\delta \rightarrow 0$,

where $1<q \leq 2$ satisfies $1 / q+1 / p=1$.

The requirement that $f_{\delta}(\cdot)$ is monotonically decreasing in Hypothesis 2 reflects the idea that the ability of a point $z \in \mathscr{B}$ to affect the local stored energy at a point $x \in \mathscr{B}$ decreases as the distance between the two points increases. The parameter $\delta \in(0, \infty)$ is a measure of the localization of the influence; a particular choice for the influence function should reflect the view that for small $\delta$, a large percentage of the mass of the function $f_{\delta}(\cdot)$ is concentrated at the origin. Figure 2 illustrates a possible influence function that is compatible with this hypothesis. This is the essence of statement (iii) in Hypothesis 2.

To more clearly distinguish the difference between the local and nonlocal parts of the total stored energy functional, it is instructive to introduce an alternative form of (3). For this, we let $f_{\delta}(\cdot)$ be as in Hypothesis 2, define

$$
a_{\delta}(x):=\int_{\mathscr{B}} f_{\delta}(x-z) d z
$$

and suppose that $e(\cdot) \in L^{p}(\mathscr{B})$. Then a simple application of the Fubini-Tonelli 
theorem and the evenness of $f_{\delta}(\cdot)$ allows us to rewrite $E_{\delta}(\cdot)$ as

$$
E_{\delta}(u)=\int_{\mathscr{B}}\left\{W(e(x))-a_{\delta}(x) e^{2}(x)+\frac{1}{2} \int_{\mathscr{B}} f_{\delta}(x-z)(e(x)-e(z))^{2} d z\right\} d x .
$$

The spatial kernel in (6) now is clearly seen to be zero on any strain field that is constant. In fact, the nonlocal convolution in (6) is exactly that portion of the energy functional that penalizes the total stored energy for strain fields that exhibit nonconstant behavior. Moreover, if $u(\cdot) \in W^{1, p}(\mathscr{B})$ is given, independent of $\delta$, then it follows from the total stored energy (3) and Hypothesis 2, (ii) and (iii), that

$$
\lim _{\delta \rightarrow 0} E_{\delta}(u)=\int_{\mathscr{B}} W\left(u^{\prime}(x)\right)-\bar{a} u^{\prime}(x)^{2} d x
$$

and

$$
\lim _{\delta \rightarrow \infty} E_{\delta}(u)=\int_{\mathscr{B}} W\left(u^{\prime}(x)\right) d x .
$$

We wish to consider the following fixed displacement minimization problem: Given $\Delta \in \mathbb{R}^{+}$and $p \in[2, \infty)$, determine $u_{0}(\cdot) \in \mathscr{A}_{>}^{1, p}(\mathscr{B})$ such that

$$
E_{\delta}\left(u_{0}\right)=\min _{u \in \mathscr{A}_{>}^{1, p}(\mathscr{B})} E_{\delta}(u) \text {, }
$$

where

$$
\mathscr{A}_{>}^{1, p}(\mathscr{B}):=\left\{u(\cdot) \in W^{1, p}(\mathscr{B}): \int_{\mathscr{B}} u^{\prime}(x) d x=\Delta \text { and } e(x)>-1 \text { for a.e. } x \in \mathscr{B}\right\},
$$

and $W^{1, p}(\mathscr{B})$ is the usual Sobolev space of functions such that for some $p \in[2, \infty)$, both the function $u(\cdot)$ and its generalized derivative $u^{\prime}(\cdot)=e(\cdot)$ are elements of $L^{p}(\mathscr{B})$. We say that a displacement field $u(\cdot): \mathscr{B} \rightarrow \mathbb{R}$ is admissible if $u(\cdot) \in$ $\mathscr{A}_{>}^{1, p}(\mathscr{B})$. In order to assure that there exists a minimizing sequence for (9) that possesses a weak limit in $\mathscr{A}_{>}^{1, p}(\mathscr{B})$, we must make two assumptions. The first is a coercivity hypothesis on the total stored energy functional $E_{\delta}(\cdot)$. This hypothesis provides for a uniform bound on any minimizing sequence $\left\{u_{n}\right\}_{n \in \boldsymbol{N}} \subset W^{1, p}(\mathscr{B})$, and thereby ensures that such a sequence is precompact in $W^{1, p}(\mathscr{B})$. In that case, there is a subsequence $\left\{u_{n_{k}}\right\}_{k \in N}$ that converges weakly to some $u_{0}(\cdot) \in W^{1, p}(\mathscr{B})$ [5, 9]. Specifically, we require $E_{\delta}(\cdot)$ to satisfy

Hypothesis 3 (Coercivity). $\exists p \in[2, \infty), \sigma_{1}>0$ and $d_{1} \in \mathbb{R}$ such that $\forall e \in \mathbb{R}$

$$
W(e)-\beta|e|^{2} \geq \sigma_{1}|e|^{p}+d_{1},
$$

where

$$
\beta:=\sup _{x \in \mathscr{B}} a_{\delta}(x) .
$$

In order to guarantee that a solution of (9) is also locally invertible (i.e., $e(x)>$ -1 for a.e. $x \in \mathscr{B})$, and consequently residing in $\mathscr{A}_{>}^{1, p}(\mathscr{B})$, we also assume the following hypothesis. 
Hypothesis 4 (Stiffness). The bulk energy function $W(\cdot): \mathbb{R} \rightarrow \mathbb{R}^{+} \cup\{\infty\}$ has the form

$$
W(e)=\left\{\begin{array}{ccc}
W(e) \in \mathbb{R}^{+} & : & e \in(-1, \infty) \\
\infty & : & e \in(-\infty,-1]
\end{array}\right\},
$$

where, in particular,

$$
\lim _{e \downarrow-1} W(e)=-\lim _{e \downarrow-1} W^{\prime}(e)=\infty .
$$

The stiffness hypothesis penalizes a deformation $y(\cdot)$ by assigning an infinite amount of bulk energy when distinct points in the reference configuration $\mathscr{B}$ occupy identical positions in the deformed configuration $y(\mathscr{B})$. Therefore, if $W(\cdot)$ satisfies Hypothesis 4 , any displacement field $u(\cdot) \in W^{1, p}(\mathscr{B})$ that satisfies $\left|E_{\delta}(u)\right|<\infty$, necessarily has the property that $u^{\prime}(x)>-1$ for a.e. $x \in \mathscr{B}$. In the remainder of this section, we require $W(\cdot)$ to have the form as given in (12).

The question of existence of a solution to (9) is difficult to answer if the bulk energy $W(\cdot)$ is nonconvex. This is because, in this case, the first portion of the energy functional (3) is not generally weakly lower-semicontinuous (w.l.s.c.) on $W^{1, p}(\mathscr{B})$. However, since $f_{\delta}(\cdot) \in L^{q}(\mathscr{B})$, it follows from slight modifications of known theorems $[5,9,14]$, that the nonlocal portion of $E_{\delta}(\cdot)$ is weakly continuous on $W^{1, p}(\mathscr{B})$. This observation, and an awareness of classical approaches in existence theory [5, 7], directs us to consider the auxiliary relaxed problem. Let

$$
W^{*}(e):=\sup \{g(e): g(e) \leq W(e) \quad \forall e \in \mathbb{R} \text { and } g(\cdot) \text { is convex }\},
$$

and define

$$
E_{\delta}^{*}(u):=\int_{\mathscr{B}}\left\{W^{*}(e(x))-e(x) \int_{\mathscr{B}} f_{\delta}(x-z) e(z) d z\right\} d x
$$

The relaxed problem associated with $(9)$ is then to determine $u_{0} \in \mathscr{A}_{>}^{1, p}(\mathscr{B})$ such that

$$
E_{\delta}^{*}\left(u_{0}\right)=\min _{u \in \mathscr{A}_{>}^{1, p}(\mathscr{B})} E_{\delta}^{*}(u)
$$

Regarding (15), since $W^{*}(\cdot)$ is convex, the local portion of (14) is now w.l.s.c. on $W^{1, p}(\mathscr{B})[5,9]$, and therefore, the relaxed energy $E_{\delta}^{*}(u)$ is as well. Consequently, we may prove [9] the following

Theorem 1 (Relaxed Existence). ${ }^{2}$ Let (4), (11), and (12) hold for some $p \in$ $[2, \infty)$ and let $f_{\delta}(\cdot) \in L^{q}(I R)$ satisfy Hypothesis 2 . Then,

$$
\inf _{u \in \mathscr{O})_{>}^{1, p}(\mathscr{B})} E_{\delta}^{*}(u)=: l_{p}^{*}=l_{p}:=\inf _{u \in \mathscr{O}_{>}^{1, p}(\mathscr{B})} E_{\delta}(u) \in \mathbb{R}
$$

\footnotetext{
${ }^{2}$ We note that the hypothesis on $f_{\delta}(\cdot)$ is only a sufficient condition; it may be weakened. In particular, aside from evenness, all that is needed from Hypothesis 2 is that $f_{\delta}(\cdot) \in L^{q}(\mathbb{R}) \cap L^{1}(\mathbb{R})$. Moreover, if we relax definition (10) to include noninvertible deformations, replacing $\mathscr{A}_{>}^{1, p}(\mathscr{B})$ by

$$
\mathscr{A}^{1, p}(\mathscr{B}):=\left\{u(\cdot) \in W^{1, p}(\mathscr{B}): \int_{\mathscr{B}} u^{\prime}(x) d x=\Delta\right\},
$$
}

then Theorem 1 also holds as stated, provided we drop the stiffness Hypothesis 4 . 
(ii) $\exists u_{0}(\cdot) \in \mathscr{A}_{>}^{1, p}(\mathscr{B})$, and a sequence $u_{k}(\cdot) \in \mathscr{A}_{>}^{1, p}(\mathscr{B})$ such that

(a) $u_{k} \rightarrow u_{0}$ in $W^{1, p}(\mathscr{B})$,

(b) $E_{\delta}^{*}\left(u_{0}\right)=\lim _{k \rightarrow \infty} E_{\delta}\left(u_{k}\right)=l_{p}$.

The derivation of the classical Euler-Lagrange equations as necessary conditions for problems (9) and (15) becomes a nontrivial matter when the stiffness Hypothesis 4 is assumed. Two difficulties are encountered in the classical derivation. The first is that given a function $u(\cdot) \in \mathscr{A}_{>}^{1, p}(\mathscr{B})$ and a possible variation $u(\cdot, \epsilon):=u(\cdot)+\epsilon \eta(\cdot)$ for $\eta(\cdot) \in W^{1, \infty}(\mathscr{B})$, it is not immediate that $u(\cdot, \epsilon) \in \mathscr{A}_{>}^{1, p}(\mathscr{B})$ when $\epsilon$ is sufficiently small. In particular, it is not clear that $e(\cdot, \epsilon)>-1$ for a.e. $x \in \mathscr{B}$ holds for $\epsilon \rightarrow 0$. Fortunately, this problem can be circumvented with a clever choice of variations [1]. The second problem is more serious. Since we do not know that $e(\cdot)$ is bounded below away from -1 , there is no reason to believe that $W^{\prime}(e(\cdot, \epsilon)) \epsilon$ $L^{1}(\mathscr{B})$ as $\epsilon \rightarrow 0$. This presents a serious obstacle to the necessary step of interchanging the differentiation of (3) with respect to $\epsilon$ and the first integral operation. For the purely local problem with no spatial dependence, this difficulty was circumvented in [1,2], and in [9] we determine necessary and sufficient conditions, similar to those in [1], to ensure that this process may be undertaken. These conditions are given in the following

Theorem 2 (Euler-Lagrange). ${ }^{3}$ Let (4) and (12) hold for some $p \in[2, \infty)$. Let $f_{\delta}(\cdot)$ satisfy Hypothesis 2, and assume that $\exists M, C>0$ such that $\left|W^{\prime}(e)\right| \leq$ $C|e|^{p}, \forall e \in[M, \infty)$. Suppose $u_{0}(\cdot) \in \mathscr{A}_{>}^{1, p}(\mathscr{B})$ is such that $E_{\delta}\left(u_{0}\right)=l_{p}$. Then, the following three conditions are equivalent:

(i) $\exists \epsilon_{0}>0$ such that

$$
\int_{\left\{-1<u_{0}^{\prime}<-1+\epsilon_{0}\right\}}\left|W^{\prime}\left(u_{0}^{\prime}(x)\right)\right| d x<\infty,
$$

(ii) $\exists \sigma \in \mathbb{R}$ such that for a.e. $x \in \mathscr{B}$

$$
W^{\prime}\left(e_{0}(x)\right)-2 \int_{\mathscr{B}} f_{\delta}(x-z) e_{0}(z) d z=\sigma,
$$

(iii) ess $\inf _{x \in \mathscr{B}} e_{0}(x)>-1$ and ess $\sup _{x \in \mathscr{B}} e_{0}(x)<\infty$.

Observe that if the hypotheses of Theorem 2 hold and $u_{0}(\cdot)$ is a solution of either (9) or (15), then, because of $(16), e_{0}(\cdot)$ must necessarily be nonconstant. This property is an immediate and obvious departure from solutions of problem (1) when the energy functional is purely local, even including higher-order gradients $[3,6,8]$.

\footnotetext{
${ }^{3}$ As in Theorem 1, the stated hypotheses may be relaxed. Specifically, regarding (4), (12), and Hypothesis 2 , we essentially need $f_{\delta}(\cdot) \in L^{q}(\mathbb{R}) \cap L^{1}(\mathbb{R})$, and $W(\cdot): \mathbb{R} \rightarrow \mathbb{R} \cup\{\infty\}$ to be continuously differentiable to guarantee the truth of the theorem. Also, if we relax definition (10) to include noninvertible deformations, replacing $\mathscr{A}_{>}^{1, p}(\mathscr{B})$ by $\mathscr{A}^{1, p}(\mathscr{B})$ (see footnote in Theorem 1), then conclusion (iii) 1 does not apply and conclusions (ii) and (iii) 2 in Theorem 2 follow as direct implications of the stated hypothesis, except that in the formal statement of Theorem 2 we should require $\left|W^{\prime}(e)\right| \leq C|e|^{p}$ for every $|e| \in[M, \infty)$, and exclude (12).
} 
In these works, it is shown that when $\Delta / l \notin[a, b]$, the global minimizer for (1) is a constant.

In solving (9), we have found [9] that a further necessary condition for minimizers of $E_{\delta}(\cdot)$ results as a direct consequence of (i) in Theorem 1:

Corollary 1 (Weierstrass Condition). Let $u_{0}(\cdot)$ be as in Theorem 1 and suppose that the hypotheses of that theorem hold. Then, for a.e. $x \in \mathscr{B}, \forall \lambda \in$ $(0,1)$ and $\forall \epsilon_{1}, \epsilon_{2} \in \mathbb{R}$ such that $e_{0}(x)=\lambda \epsilon_{1}+(1-\lambda) \epsilon_{2}$,

$$
W\left(e_{0}(x)\right) \leq \lambda W\left(\epsilon_{1}\right)+(1-\lambda) W\left(\epsilon_{2}\right) .
$$

In particular, the Weierstrass condition requires that any solution of (9) must possess a strain field $e_{0}(\cdot)$ whose range nontrivially intersects $(-1, a] \cup[b, \infty)$ almost everywhere in the body $\mathscr{B}$ (see Figs. la and $1 \mathrm{~b}$ ). This places a restriction on the type of strain discontinuities a solution $u_{0}(\cdot)$ may support. These restrictions are called jump conditions and, for simple discontinuities, are summarized in

Corollary 2 (Weierstrass Jump Conditions). Let the hypotheses of Theorem 2 and Corollary 1 be satisfied. Suppose that $x_{0} \in \mathscr{B}$ is such that

$$
e_{0}\left(x_{0}-0\right)=e_{1} \neq e_{2}=e_{0}\left(x_{0}+0\right)
$$

holds. Then,

(i) $W^{\prime}\left(e_{1}\right)=W^{\prime}\left(e_{2}\right)=: \bar{\sigma}$,

(ii) $\bar{\sigma}\left(e_{1}-e_{2}\right)=W\left(e_{1}\right)-W\left(e_{2}\right)$.

This corollary implies that a simple discontinuity in a minimizing strain field must occur at the Maxwell stress, denoted by $\bar{\sigma}$ here and in Figs. $1 \mathrm{a}$ and $1 \mathrm{~b}$.

In the remainder of this paper, we shall restrict ourselves to a specific convex bulk energy $W(\cdot)$ and an exponential influence function $f_{\delta}(\cdot)$. This approach isolates the nonlocal properties of the body from any effects that could be attributed to a nonconvexity in $W(\cdot)$, and allows us to solve problem (9) explicitly. We believe that this opens a clear pathway to understanding the fundamental effects of nonlocal spatial dependence.

2. The reduced model: A necessary differential equation. We now focus on the minimization problem (9) in the case that the bulk energy $W(\cdot)$ is a positive-definite quadratic form with minimum at zero. We also assume an exponential form for the nonlocal influence function $f_{\delta}(\cdot)$ that satisfies Hypothesis 2 . Thus, in the remainder of this work we take $W(e)=k e^{2}$, where $k>0$, and define

$$
f_{\delta}(\eta)=\frac{a}{\delta} e^{-|\eta| / \delta}
$$

for all $\eta \in \mathbb{R}$, and nonzero $a, \delta \in \mathbb{R}^{+}$. Since $W(\cdot)$ does not exhibit any singular behavior at $x=-1$, we will take the class of admissible displacement fields for the minimization problem (9) to be as in (10) except that the condition of $e(x)>-1$ for a.e. $x \in \mathscr{B}$ is omitted. In this case, the stiffness hypothesis (12) is not relevant, and in the remainder of this work we replace (9) by the following 
minimization problem: For $p \in[2, \infty)$, determine $u_{0}(\cdot) \in \mathscr{A}^{1, p}(\mathscr{B})$ such that

$$
E_{\delta}\left(u_{0}\right)=\min _{u \in \mathscr{A}^{1 \cdot p}(\mathscr{B})} E_{\delta}(u)
$$

where

$$
\mathscr{A}^{1, p}(\mathscr{B}):=\left\{u(\cdot) \in W^{1, p}(\mathscr{B}): \int_{\mathscr{B}} u^{\prime}(x) d x=\Delta\right\} .
$$

Since $\left|W^{\prime}(e)\right| \leq 2 k e^{2}$ for every $|e| \geq 1$, and $f_{\delta}(\cdot) \in L^{q}(\mathbb{R})$ for every $q \in(1, \infty)$, Theorem 2 then implies that if $u(\cdot) \in \mathscr{A}^{1, p}(\mathscr{B})$ is a solution of (19) for $p=2$, then $u(\cdot) \in \mathscr{A}^{1, \infty}(\mathscr{B})$ and there exists $\sigma \in \mathbb{R}$ such that the following Euler-Lagrange integral equation holds:

$$
T(x):=2 k e(x)-2 \int_{\mathscr{B}} f_{\delta}(x-z) e(z) d z=\sigma \text { for a.e. } x \in \mathscr{B} .
$$

It is clear that any solution $u(\cdot)$ of (19) may be adjusted on a set of Lebesgue measure zero so that it still solves (19) and satisfies (21) for every point $x \in \mathscr{B}$. Thus, for all $x \in \mathscr{B}$, it follows that

$$
e(x)=\frac{1}{k} \int_{0}^{x} f_{\delta}(x-z) e(z) d z+\frac{1}{k} \int_{x}^{l} f_{\delta}(x-z) e(z) d z+\frac{\sigma}{2 k},
$$

and because of the form of $f_{\delta}(\cdot)$ in (18), we see that $u(\cdot)$ must be at least of class $C^{3}(\mathscr{B})$, thereby justifying the following argument: Observe that by differentiating (21) and use of (18) we readily obtain

$$
T^{\prime}(x)=2 k e^{\prime}(x)+\frac{2}{\delta} \int_{0}^{x} f_{\delta}(x-z) e(z) d z-\frac{2}{\delta} \int_{x}^{l} f_{\delta}(x-z) e(z) d z .
$$

Again, by use of $(21)$ we see that

$$
T^{\prime}(x)+\frac{1}{\delta} T(x)=2 k e^{\prime}(x)+\frac{1}{\delta} 2 k e(x)-\frac{4}{\delta} \int_{x}^{l} f_{\delta}(x-z) e(z) d z
$$

and

$$
T^{\prime}(x)-\frac{1}{\delta} T(x)=2 k e^{\prime}(x)-\frac{1}{\delta} 2 k e(x)+\frac{4}{\delta} \int_{0}^{x} f_{\delta}(x-z) e(z) d z .
$$

Finally, by adding (23) and (24) together and differentiating once more, we find

$$
\frac{d}{d x}\left(T^{\prime}(x)\right)=2 k e^{\prime \prime}(x)+\frac{4}{\delta} f_{\delta}(0) e(x)-\frac{2}{\delta^{2}} \int_{0}^{l} f_{\delta}(x-z) e(z) d z,
$$

which, because of (18), (21), and the fact that $T \equiv \sigma$, produces the following ordinary differential equation for $e(\cdot)$ :

$$
e^{\prime \prime}(x)+\frac{1}{k}\left(\frac{2 a-k}{\delta^{2}}\right) e(x)=-\frac{\sigma}{2 k \delta^{2}} .
$$

This ordinary differential equation represents a necessary condition on any solution of (21), and has a two-parameter family of solutions. These parameters combine with $\sigma$ to give three free constants at this stage in the solution to the minimization 
problem (19). In the next section, this nonuniqueness will be removed by returning to the original Euler-Lagrange equation (21) to gain sufficient conditions for its solvability. Of course, it is also essential to note that any solution $u(\cdot) \in \mathscr{A}^{1, \infty}(\mathscr{B})$ of (21) is required to satisfy the fixed displacement condition

$$
\int_{\mathscr{B}} e(x) d x=\Delta .
$$

If we apply the standard Taylor expansion argument of "approximation", as was proposed and advanced by van der Waals [12], to the nonlocal part of the functional (6) we are guided to replace the functional in (6) by the gradient model

$$
E_{\delta}^{\#}(u):=\int_{\mathscr{B}}\left\{W(e(x))-a_{\delta}(x) e^{2}(x)+b_{\delta}(x)\left[e^{\prime}(x)\right]^{2}\right\} d x
$$

at the lowest nontrivial order of approximation, where

$$
b_{\delta}(x):=\frac{1}{2} \int_{\mathscr{B}}(z-x)^{2} f_{\delta}(x-z) d z .
$$

If $\delta$ is sufficiently small it can be shown from (5), (18), and (29) that $a_{\delta}(x) \simeq 2 a$ and $b_{\delta}(x) \simeq 2 a \delta^{2}$ except near the boundary of $\mathscr{B}$. In any case, for $W(e)=k e^{2}$, the Euler-Lagrange equation associated with the minimization of (28) subject to the constraint (27) is a regular second-order ordinary differential equation for $e(x)$ containing an unknown Lagrange multiplier constant. The natural boundary conditions will require $e^{\prime}(x)=0$ on $\partial \mathscr{B}$, and these, along with (27), will determine the three constants of this theory. Since the more complete nonlocal theory does not require $e^{\prime}(x)$ to vanish at the boundary $\partial \mathscr{B}$, we expect that at least in a neighborhood of $\partial \mathscr{B}$ the "approximation" will break down. Moreover, if $W(\cdot)$ is nonconvex, there is strong evidence [10] to suggest that similar breakdowns of the boundary layer type occur even in the interior of $\mathscr{B}$. Later, we give examples of the nonlocal theory for convex $W(\cdot)$ which show that large strain gradients do occur at the boundary $\partial \mathscr{B}$. If, in (28), $a_{\delta}(x)$ and $b_{\delta}(x)$ are simply replaced by constants then for convex $W(\cdot)$ the strain field that minimizes (28) subject to (27) is constant.

3. The structure of admissible extremals. The differential equation introduced in (26) has solutions whose form depends on the sign of the expression $2 a-k$. Here, we show that in each of the three cases $k>2 a, k=2 a$, and $k<2 a$, the solutions of (26) are compatible with the Euler-Lagrange equation (21), if and only if a certain $2 \times 3$ system of linear equations for three arbitrary constants is satisfied. These equations, coupled with the fixed displacement condition (27), yield a $3 \times 3$ linear system whose solutions are guaranteed to solve the Euler-Lagrange equation (21).

For the remainder of this work, it is convenient to introduce the material parameter $\omega>0$ defined by

$$
\omega^{2} \equiv\left|\frac{k-2 a}{k \delta^{2}}\right|
$$

and to let $c_{1}, c_{2} \in \mathbb{R}$ denote the arbitrary constants that are contained in the general solution of (26). 
Case (1) $k>2 a$. Here, (26) implies that

$$
e(x)=c_{1} e^{\omega x}+c_{2} e^{-\omega x}+\frac{\sigma}{2(k-2 a)},
$$

and this, with (18) and (21), yields

$$
\begin{aligned}
T(x)= & \sigma+\left(\frac{2 a c_{1}}{1+\omega \delta}+\frac{2 a c_{2}}{1-\omega \delta}+\frac{a \sigma}{k-2 a}\right) e^{-x / \delta} \\
& +\left(\frac{2 a c_{1}}{1-\omega \delta} e^{\omega l}+\frac{2 a c_{2}}{1+\omega \delta} e^{-\omega l}+\frac{a \sigma}{k-2 a}\right) e^{(x-l) / \delta} \\
= & \sigma .
\end{aligned}
$$

Here, we note that $\omega \delta-1 \neq 0$ since $a>0$. Now, since (32) must hold for every $x \in \mathscr{B}$, it follows that the coefficients of $e^{-x / \delta}$ and $e^{(x-l) / \delta}$ must vanish. This provides two of the three equations we are seeking, and the fixed displacement condition (27), when applied to (31), yields the third. Thus, we have the following linear system for determining $c_{1}, c_{2}$, and $\sigma$ :

$$
\begin{aligned}
\frac{2 a}{1+\omega \delta} c_{1}+\frac{2 a}{1-\omega \delta} c_{2}+\frac{a}{k-2 a} \sigma & =0, \\
\frac{2 a e^{\omega l}}{1-\omega \delta} c_{1}+\frac{2 a e^{-\omega l}}{1+\omega \delta} c_{2}+\frac{a}{k-2 a} \sigma & =0, \\
\frac{e^{\omega l}-1}{\omega} c_{1}+\frac{1-e^{-\omega l}}{\omega} c_{2}+\frac{l}{2(k-2 a)} \sigma & =\Delta .
\end{aligned}
$$

Any solution $\left(c_{1}, c_{2}, \sigma\right)$ of this system, when coupled with (31), necessarily solves (21) and the associated fixed displacement condition (27).

Case (2) $k=2 a$. When $k=2 a$, (26) reduces to a trivial form which has the general solution

$$
e(x)=-\frac{\sigma}{4 k \delta^{2}} x^{2}+c_{1} x+c_{2} .
$$

Upon substituting (34) and (18) into (21) and simplifying as in Case (1) above, we get

$$
\begin{aligned}
T(x)= & \sigma+\left(-2 a \delta c_{1}+2 a c_{2}-\frac{\sigma a}{k}\right) e^{-x / \delta} \\
& +\left((2 a l+2 a \delta) c_{1}+2 a c_{2}-\frac{\sigma a}{2 k \delta^{2}}\left(l^{2}+2 \delta l+2 \delta^{2}\right)\right) e^{(x-l) / \delta} \\
= & \sigma .
\end{aligned}
$$

Again, by recognizing that the coefficients of $e^{-x / \delta}$ and $e^{(x-l) / \delta}$ in (35) must vanish, and by applying the fixed displacement condition (27), we find the following linear 
system for determining $c_{1}, c_{2}$, and $\sigma$ :

$$
\begin{aligned}
-2 a \delta c_{1}+2 a c_{2}-\frac{a}{k} \sigma & =0, \\
(2 a l+2 a \delta) c_{1}+2 a c_{2}-\frac{\sigma a}{2 k \delta^{2}}\left(l^{2}+2 \delta l+2 \delta^{2}\right) & =0, \\
\frac{l^{2}}{2} c_{1}+l c_{2}-\frac{l^{3}}{12 k \delta^{2}} \sigma & =\Delta .
\end{aligned}
$$

Any solution $\left(c_{1}, c_{2}, \sigma\right)$ of this system, when coupled with (34), necessarily solves (21) and the associated fixed displacement condition (27).

Case (3) $k<2 a$. Proceeding as before, we see that when $k<2 a$, the general solution of $(26)$ is

$$
e(x)=c_{1} \sin \omega x+c_{2} \cos \omega x+\frac{\sigma}{2(k-2 a)} .
$$

Upon substituting (37) and (18) into (21) and performing a significant amount of tedious integration and simplification, we get

$$
\begin{aligned}
T(x)=\sigma & +\left(k c_{1}-k \omega \delta c_{2}+\frac{a}{(k-2 a)} \sigma\right) e^{-x / \delta} \\
& +(k \cos \omega l-k \delta \omega \sin \omega l) c_{1} e^{(x-l) / \delta} \\
& +\left((k \sin \omega l+k \omega \delta \cos \omega l) c_{2}+\frac{a}{(k-2 a)} \sigma\right) e^{(x-l) / \delta} \\
=\sigma &
\end{aligned}
$$

As in the previous two cases, it follows that the coefficients of $e^{-x / \delta}$ and $e^{(x-l) / \delta}$ in (38) must vanish. This, together with the fixed displacement condition (27) applied to (37), then gives the following system for determining $c_{1}, c_{2}$, and $\sigma$ :

$$
k c_{1}-k \omega \delta c_{2}+\frac{a}{(k-2 a)} \sigma=0,
$$

$(k \cos \omega l-k \delta \omega \sin \omega l) c_{1}$

$$
\begin{aligned}
+(k \sin \omega l+k \omega \delta \cos \omega l) c_{2}+\frac{a}{(k-2 a)} \sigma & =0, \\
& \frac{\sin \omega l}{\omega} c_{1}+\frac{1-\cos \omega l}{\omega} c_{2}+\frac{l}{2(k-2 a)} \sigma=\Delta .
\end{aligned}
$$

As in the Cases (1) and (2), any solution of this system, when coupled with (37), necessarily solves the Euler-Lagrange equation (21) and the fixed displacement condition (27).

For easy reference, it will be convenient to denote the coefficient matrices of the linear systems (33), (36), and (39) for the Cases (1), (2), and (3), respectively, as $A_{i}$ where $i=1,2,3$. Then, by defining

$$
\mathbf{d}:=\left(\begin{array}{c}
0 \\
0 \\
\Delta
\end{array}\right) \quad, \quad \mathbf{c}:=\left(\begin{array}{c}
c_{1} \\
c_{2} \\
\sigma
\end{array}\right),
$$


the relevant linear systems for the respective Cases (1), (2), and (3) may be written in the compact form

$$
A_{i} \mathbf{c}=\mathbf{d} \text { for } i=1,2,3 .
$$

In view of (41), if we wish to exhibit a unique solution of (21) and (27), then we must be assured of the existence of the inverse matrices $A_{i}^{-1}$ for $i=1,2,3$. In the next section, we see, unequivocally, that $A_{i}^{-1}$ exists for the Cases (1) and (2), i.e., $i=1,2$. However, some interesting existence and stability questions arise for the Case (3), i.e., $i=3$. These issues will be considered in Sec. 5 .

4. Analysis of minimizers in the Cases (1) and (2): $k \geq 2 a$. In this section we study the existence and uniqueness of solutions to the minimization problem (19) for the Cases (1) and (2). To begin, we note that in these two cases the coercivity hypothesis (11) is satisfied, for $p=2$, by the stored energy functional (3) with $W(e)=k e^{2}$ and $f_{\delta}(\cdot)$ given by (18). To see this, we first observe that (5) and (18) yield

$$
a_{\delta}(x)=2 a-a\left(e^{-x / \delta}+e^{(x-l) / \delta}\right)
$$

Thus,

$$
k-a_{\delta}(x)=(k-2 a)+a\left(e^{-x / \delta}+e^{(x-l) / \delta}\right),
$$

and since $a\left(e^{-x / \delta}+e^{(x-l) / \delta}\right)$ is bounded below away from zero on $\mathscr{B}$, it follows that for $W(e)=k e^{2}$ the coercivity hypothesis (11) holds for $p=2$. Further, since $W(\cdot)$ is convex and $f_{\delta}(\cdot) \in L^{q}(\mathscr{B})$ for every $q \in(1, \infty)$, Theorem 1 implies that there exists a solution $u(\cdot) \in \mathscr{A}^{1,2}(\mathscr{B})$ of the minimization problem (19) if $k \geq 2 a$. Theorem 2 then implies that $u(\cdot) \in \mathscr{A}^{1, \infty}(\mathscr{B})$, and also that $u(\cdot)$ satisfies the EulerLagrange equation (21); thus it must have one of the smooth forms generated in Sec. 3. To determine whether any of these forms actually solves the minimization problem (19) for Cases (1) and (2), we must then solve (41) for $i=1,2$. From Theorem 2, we note that since any solution $u(\cdot) \in \mathscr{A}^{1, p}(\mathscr{B})$ of $(19)$ must necessarily reside in $\mathscr{A}^{1, \infty}(\mathscr{B})$, and $\mathscr{A}^{1, \infty}(\mathscr{B}) \subset \mathscr{A}^{1, p}(\mathscr{B})$ when $p \in(0, \infty)$, then $l_{p}=l_{\infty}$ for every $p \in[2, \infty)$. Therefore, any solution $u(\cdot)$ of $(19)$ for $p=2$ actually solves (19) for every $p \in[2, \infty]$. Thus, for the remainder of this work we will assume without loss of generality that $p \in[2, \infty]$, keeping in mind that our initial analysis required $p=2$.

Case (1) $k>2 a(i=1)$. In this case, a straightforward calculation using $A_{1}$ as defined in (41), and inferred from (33), gives

$$
\begin{aligned}
\operatorname{det} A_{1}= & 2 a^{2}\left(-1+e^{l \omega}+\delta \omega+e^{l \omega} \delta \omega\right) \\
& \times\left\{\frac{\left(2-2 e^{l \omega}+l \omega+e^{l \omega} l \omega-l \delta \omega^{2}\right)}{e^{l \omega}(2 a-k) \omega(-1+\delta \omega)^{2}(1+\delta \omega)^{2}}\right. \\
& \left.+\frac{\left(e^{l \omega} l \delta \omega^{2}-2 \delta^{2} \omega^{2}+2 e^{l \omega} \delta^{2} \omega^{2}\right)}{e^{l \omega}(2 a-k) \omega(-1+\delta \omega)^{2}(1+\delta \omega)^{2}}\right\} .
\end{aligned}
$$


Then, because of (30) and the inequalities $a>0, k>0$, we see that $\delta \omega \neq 1$, and it follows that the denominator of (44) is strictly negative. Also, since $\omega>0$, the first term of the product in (44) is nonzero as well. Now, let $G(\omega)$ be defined as the numerator in the second term of the product in (44), and note that, equivalently,

$$
G(\omega)=\left(e^{l \omega}-1\right)\left(l \omega^{2} \delta+2 \omega^{2} \delta^{2}\right)+\psi(\omega),
$$

where $\psi(\omega):=(l \omega-2) e^{l \omega}+l \omega+2$. Then, it follows that $\psi(0)=\psi^{\prime}(0)=0$, and that $\psi^{\prime \prime}(\omega)>0$ for every $\omega>0$; thus, $\psi(\omega)>0$ on $(0, \infty)$. Combining this with the positivity of $G(\omega)-\psi(\omega)$, we see that $G(\omega)>0$ for every $\omega>0$. Therefore, the determinant of $A_{1}$ is nonzero for every $\omega>0$ and $A_{1}^{-1}$ exists and is unique for every $\omega>0$ when $k>2 a$. Thus, $\mathbf{c}=A_{1}^{-1} \mathbf{d}$ uniquely solves (41), and with (40) we conclude that (31) generates the unique solution of (19) for every $p \in[2, \infty]$. Specifically, while the computation of $A_{1}^{-1}$ is long and tedious, because of the simple structure of $\mathbf{d}$, it is straightforward to determine $\mathbf{c}$, i.e., $c_{1}, c_{2}$, and $\sigma$. We find

$$
\begin{aligned}
& c_{1}=-\frac{2 a \omega}{k G(\omega)} \Delta, \\
& c_{2}=-\frac{2 a \omega e^{l \omega}}{k G(\omega)} \Delta, \\
& \sigma=\frac{2(k-2 a) \omega\left(1+e^{l \omega}-\delta \omega+e^{l \omega} \delta \omega\right)}{G(\omega)} \Delta .
\end{aligned}
$$

When (46) is substituted into (31) and simplified, we obtain the following minimizing field for (19) in the Case (1):

$$
e_{1, \delta}(x):=-\frac{4 \omega a \Delta}{k G(\omega)} e^{l \omega / 2} \cosh \left\{\omega\left(x-\frac{l}{2}\right)\right\}+\frac{\sigma}{2(k-2 a)} .
$$

Clearly, $e_{1, \delta}(\cdot)$ is smooth and symmetric about $x=l / 2$ for all $\delta>0$, and it follows from $(30),(46)_{3}$, and (47) that $e_{1, \delta}(\cdot)>0$ on $\mathscr{B}$ if and only if $\Delta>0$.

It is of interest to consider the asymptotic behavior of (47) as $\delta \rightarrow 0$; i.e., as the spatial dependence of the material becomes more locally concentrated. To do this, we first recall from (30) that $\omega^{-1}=O(\delta)$ as $\delta \rightarrow 0$. Then, (45) yields

$$
G(\omega) \omega^{-1} e^{-\omega l}=l(1+\delta \omega)+O\left(\omega^{-1}\right),
$$

and with (30) and (46) we obtain

$$
\begin{aligned}
& c_{1}=\frac{-2 a e^{-l \omega}}{k\left(l(1+\delta \omega)+O\left(\omega^{-1}\right)\right)} \Delta, \\
& c_{2}=\frac{-2 a}{k\left(l(1+\delta \omega)+O\left(\omega^{-1}\right)\right)} \Delta, \\
& \sigma=\frac{2(k-2 a)\left(\left(1+e^{-l \omega}\right)+\delta \omega\left(1-e^{-l \omega}\right)\right)}{l(1+\delta \omega)+O\left(\omega^{-1}\right)} \Delta .
\end{aligned}
$$


Then, using (31), we find

$$
e_{1,0}(x):=\lim _{\delta \rightarrow 0} e_{1, \delta}(x)=\left\{\begin{array}{ccc}
\frac{\Delta}{l} & : & x \in(0, l) \\
\frac{\Delta}{l} \delta \omega & : & x=0, l
\end{array}\right\},
$$

which, of course, also follows from (47). We note, from (30), that the discontinuity exhibited in (50) at the boundary points is nontrivial, approaching a maximum of $\Delta / l$ as $k \downarrow 2 a$ and a minimum of 0 as $k \rightarrow \infty$. In order to manifest this behavior as $\delta \rightarrow 0$, the strain field $e_{1, \delta}(\cdot)$ of (47) supports high gradients in localized regions near $\partial \mathscr{B}$ while exhibiting nearly a constant value of $\Delta / l$ in the interior of $\mathscr{B}$ for small positive $\delta$. These high gradient boundary layers become sharply focused in the limit $\delta \rightarrow 0$ as seen in Fig. 3 .

It is straightforward to show $[9,14]$ that $f_{\delta}(\cdot)$ given in (18) satisfies Hypothesis 2 with $\bar{a}=2 a$. Therefore, using (7), for $u(\cdot) \in \mathscr{A}^{1, p}(\mathscr{B})$, we observe that

$$
E_{0}(u):=\lim _{\delta \rightarrow 0} E_{\delta}(u)=\int_{\mathscr{B}}(k-2 a) u^{\prime}(x)^{2} d x .
$$

The unique solution of the minimization problem (19) for the functional $E_{0}(\cdot)$ is well known for the case $k>2 a$; it is the constant strain field $\Delta / l[6,8]$. Thus, we conclude that the limit of $e_{1, \delta}(\cdot)$ as $\delta \rightarrow 0$ coincides with the minimizer of $E_{0}(\cdot)$ over $\mathscr{A}^{1, p}(\mathscr{B})$ everywhere except for $x \in \partial \mathscr{B}$. This assertion also holds if we replace $E_{0}(\cdot)$ with the analogous gradient model of Gurtin, Slemrod, and Carr [9].

Now, we wish to show that the asymptotic behavior of the minimizing displacement field $u_{1, \delta}(\cdot) \in \mathscr{A}^{1, p}(\mathscr{B})$ associated with (47) respects that of the functional $E_{\delta}$ in the limit as $\delta \rightarrow 0$, in the sense that

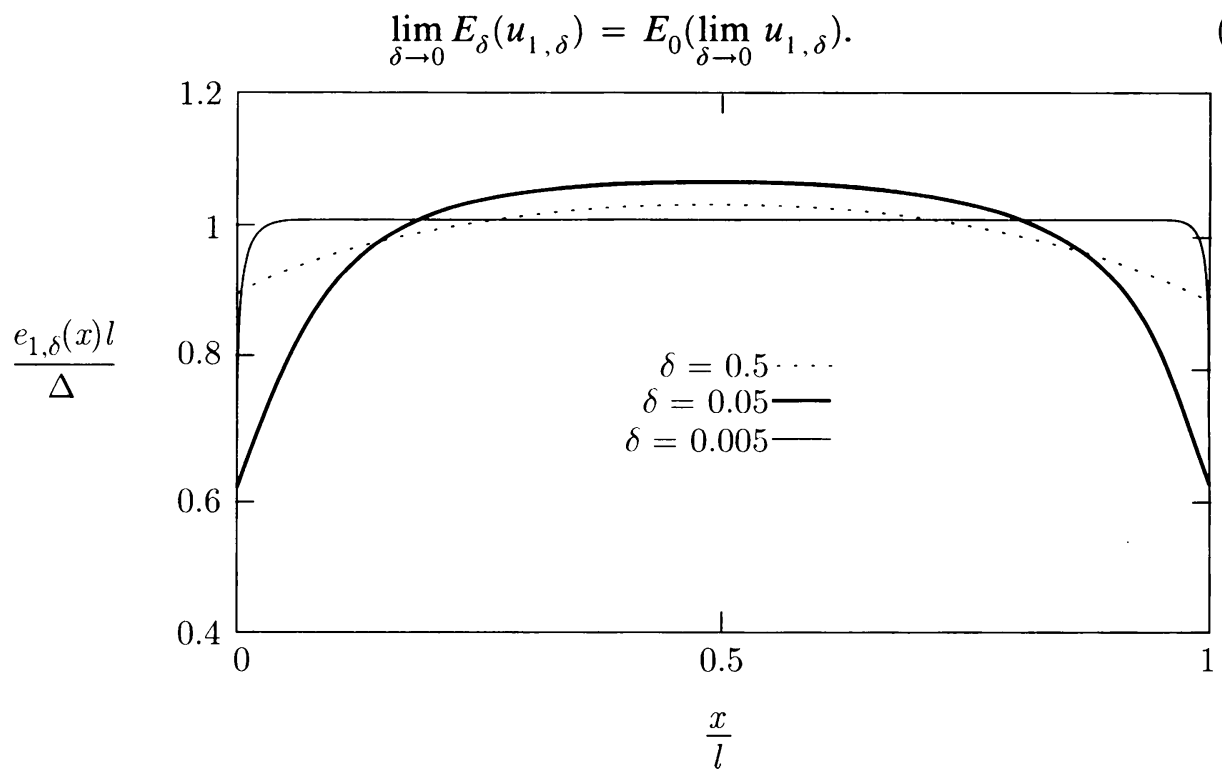

FIG. 3. The minimizing strain field of (47) for $k=3 a$ and for various values of the influence parameter $\delta$ 
To do this, we first observe, from Theorem 2 , that if $u(\cdot) \in \mathscr{A}^{1, p}(\mathscr{B})$ solves the Euler-Lagrange equation (21), we have

$$
\begin{aligned}
E_{\delta}(u) & =\int_{\mathscr{B}}\left\{k u^{\prime}(x)^{2}-u^{\prime}(x) \int_{\mathscr{B}} f_{\delta}(x-z) u^{\prime}(z) d z\right\} d x \\
& =\frac{\sigma}{2} \int_{\mathscr{B}} u^{\prime}(x) d x \\
& =\frac{1}{2} \Delta \sigma .
\end{aligned}
$$

Then, recalling $(49)_{3}$,

$$
\lim _{\delta \rightarrow 0} E_{\delta}\left(u_{1, \delta}\right)=(k-2 a) \frac{\Delta^{2}}{l},
$$

which allows us to conclude (52).

Note, from $(46)_{3}$, that for a given displacement $\Delta$ the stress $\sigma$ is linear in the gross displacement $\Delta$. If we temporarily rewrite $(46)_{3}$ as

$$
\sigma=\phi(\delta) \Delta,
$$

for a fixed $l, k$, and $a$, then it readily follows that $\partial \phi / \partial \delta>0$. Thus, for a given gross displacement $\Delta>0$, if the material is replaced by one of lesser spatial influence (i.e., if $\delta$ is decreased) then the level of stress necessary for equilibrium is decreased. We conclude that a focusing of the spatial dependence through the influence function $f_{\delta}(\cdot)$ by reducing the value of $\delta$ acts as a softening agent in the effective stiffness modulus of the material ${ }^{4}$ (see Fig. 4). The limiting value of $\sigma$ as $\delta \rightarrow 0$ is $2(k-2 a) \Delta / l$, exactly the value predicted by solving (19) for $E_{0}(\cdot)$.

In the limiting case $\delta \rightarrow \infty$, the identity approximating property of the spatial influence function $f_{\delta}(\cdot)$ is essentially nullified by (ii) of Hypothesis 2 on the spatial energy. This asymptotics identifies the limiting form of the minimizing displacement field as the effect of spatial dependence is "turned off". Analogous to the previous discussion, we find

$$
\lim _{\delta \rightarrow \infty} e_{1, \delta}(x)=\frac{\Delta}{l}
$$

and

$$
\lim _{\delta \rightarrow \infty} E_{\delta}\left(u_{1, \delta}\right)=E_{\infty}\left(\frac{\Delta}{l}\right),
$$

where, according to $(8)$, we have defined

$$
E_{\infty}(u):=\int_{\mathscr{B}} k u^{\prime}(x)^{2} d x
$$

for $u(\cdot) \in \mathscr{A}^{1, p}(\mathscr{B})$. In this case, no discontinuity appears at the boundary points of $\mathscr{B}$ in the limit. This is due to the diminishing magnitude of the nonlocal spatial term in $E_{\delta}(\cdot)$ as $\delta \rightarrow \infty$.

\footnotetext{
${ }^{4}$ Similarly, for a fixed $l, a$, and $\delta$, we have that $\partial \phi / \partial k>0$, and therefore when the material is replaced by one of greater elastic stiffness $k$ the level of stress necessary for equilibrium is increased. This hardening effect is illustrated in Fig. 4 by the uniform raising of the stress-influence graphs as $k$ is increased relative to $a$.
} 


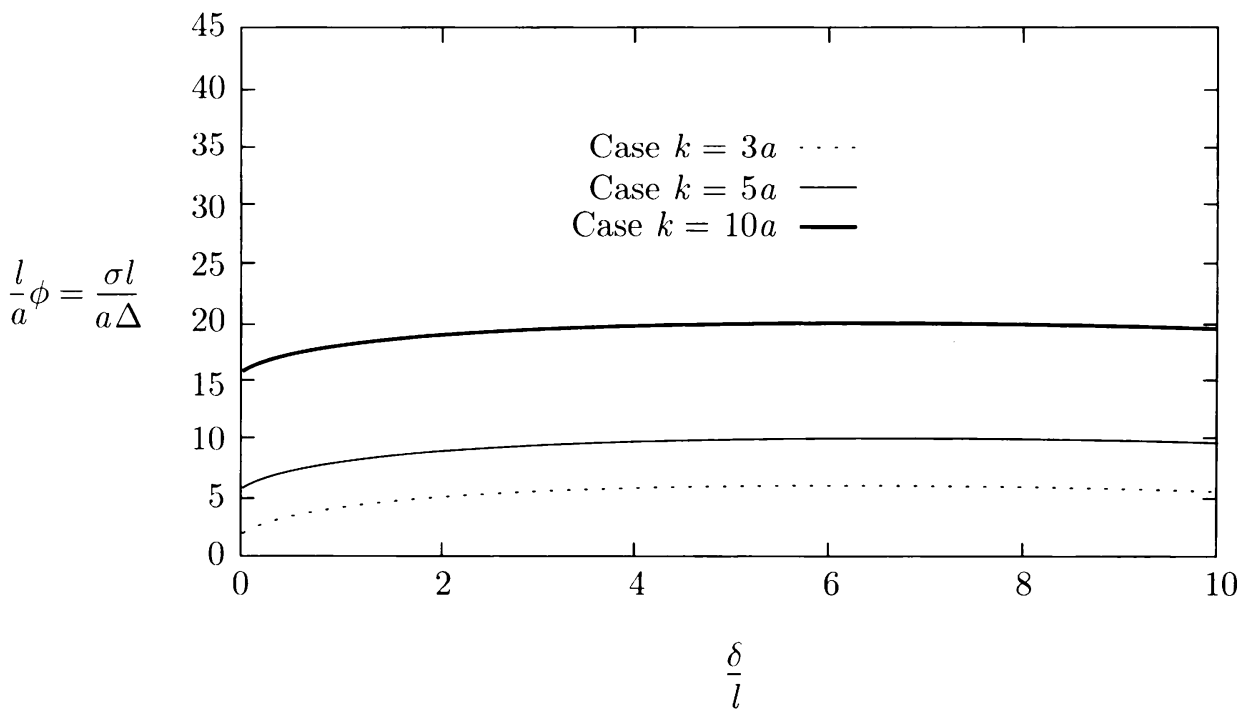

FIG. 4. The normalized effective stiffness modulus $\phi(\delta)$ of (55) for the cases $k=3 a, k=5 a$, and $k=10 a$

Case (2) $k=2 a(i=2)$. Here, we recall $A_{2}$ as defined in (41), and inferred from (33), to obtain

$$
\operatorname{det} A_{2}=-\frac{a^{2} l(l+2 \delta)\left(l^{2}+6 l \delta+12 \delta^{2}\right)}{3} .
$$

Clearly, det $A_{2} \neq 0$ and $A_{2}^{-1}$ exists and is unique for every $\delta \geq 0$. Hence, as in Case (1), $\mathbf{c}=A_{2}^{-1} \mathbf{d}$ uniquely solves (41), and with (40) we find that (34) generates the unique solution of the minimization problem (19) for every $p \in[2, \infty]$. We find that $\mathbf{c}$, i.e., $c_{1}, c_{2}$, and $\sigma$, is given by

$$
\begin{gathered}
c_{1}=\frac{6}{l^{2}+6 l \delta+12 \delta^{2}} \Delta, \\
c_{2}=\frac{6 \delta(l+2 \delta)}{l^{3}+6 l^{2} \delta+12 l \delta^{2}} \Delta, \\
\sigma=\frac{24 k \delta^{2}}{l^{3}+6 l^{2} \delta+12 l \delta^{2}} \Delta .
\end{gathered}
$$

When this is substituted into (34) and simplified we find that

$$
e_{2, \delta}(x):=\frac{12 \delta^{2}+6 l \delta+\frac{3}{2} l^{2}-6\left(x-\frac{l}{2}\right)^{2}}{l^{2}+6 l \delta+12 \delta^{2}} \frac{\Delta}{l}
$$

is the minimizing strain field for (19). Because $\Delta>0$, it is clear from (61) that $e_{2, \delta}(\cdot)>0$ on the entire body $\mathscr{B}$. As in Case (1), we note that $e_{2, \delta}(\cdot)$ is smooth, symmetric, and even about $x=l / 2$.

The asymptotics for this case behave somewhat differently than in the previous case. First, the limiting strain field in $\mathscr{B}$ as $\delta \rightarrow 0$ is not a constant field as it is in Case (1), but rather it is a positive, inverted parabola, symmetric about $x=l / 2$. 


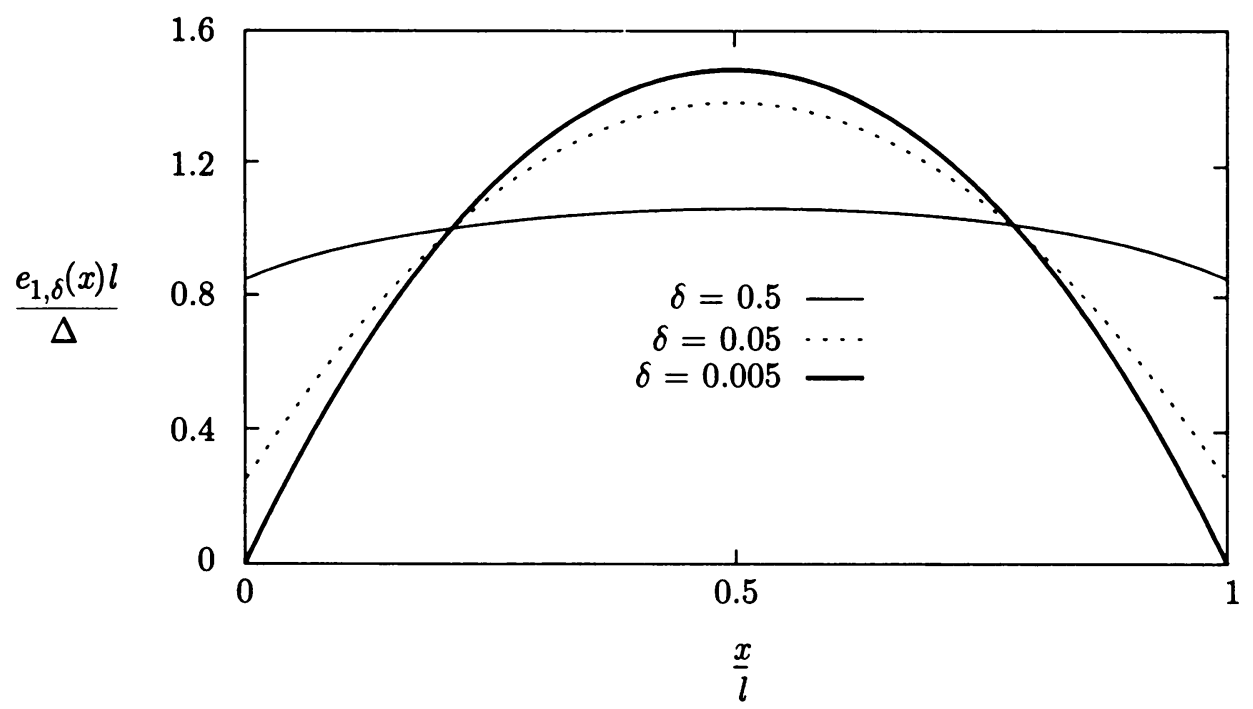

FIG. 5. The minimizing strain field of $(61)$ for $k=2 a$ and for various values of the influence parameter $\delta$

Specifically, we have from (61) that

$$
e_{2,0}(x):=\lim _{\delta \rightarrow 0} e_{2, \delta}(x)=\frac{3 l^{2}-12\left(x-\frac{l}{2}\right)^{2}}{2 l^{2}} \frac{\Delta}{l} .
$$

From (61), we see that the maximum strain for any given $\delta>0$ is achieved at $x=l / 2$ and, according to (62), this value increases to $3 \Delta /(2 l)$ at $\delta=0$. Similarly, the minimum positive strain is achieved for $x \in \partial \mathscr{B}$ and this value decreases to 0 at $\delta=0$ (see Fig. 5). Recalling (7), we note that in this case $E_{0}(u) \equiv 0$ for all $u(\cdot) \in \mathscr{A}^{1, p}(\mathscr{B})$. Thus, every displacement field $u(\cdot) \in \mathscr{A}^{1, p}(\mathscr{B})$ is a solution to the minimization problem (19) for $E_{0}(\cdot)$, including the constant solution $\Delta / l$ as in Case (1). In this case $(k=2 a)$, the effect of localizing the spatial influence by letting $\delta \rightarrow 0$ produces a negating effect on the coercivity of the functional $E_{\delta}(\cdot)$ that is induced by the bulk energy $W(\cdot)$. This competition appears to act as a selection mechanism in choosing the particular limiting strain field $e_{2,0}(\cdot)$. The fact that $E_{0}(\cdot) \equiv 0$ is respected in the energy asymptotics is seen via $(53)$ and (60), i.e.,

$$
\lim _{\delta \rightarrow 0} E_{\delta}\left(u_{2, \delta}\right)=E_{0}\left(\lim _{\delta \rightarrow 0} u_{2, \delta}\right)=0 .
$$

To continue, we observe from $(60)_{3}$ that the stress $\sigma$ is linear in $\Delta$. Moreover, similar to the behavior as found in Case (1), here we find the same softening behavior in $\mathscr{B}$ due to the localization as $\delta \rightarrow 0$ of the nonlocal influence. In this case, however, the limiting effective modulus is zero.

In the alternative limit $\delta \rightarrow \infty$ of zero spatial dependence, the limiting strain field and total stored energy are identical to those recorded in (56) and (57) for Case (1).

5. The minimization problem in the Case (3): $k<2 a$. Contrary to the straightforward analysis in Cases (1) and (2) given above, this case presents a number of difficulties to determining a minimizer of problem (19), if, in fact, one exists. The 
most pressing problem is that for $k$ sufficiently less than $2 a$, an unavoidable violation of the coercivity hypothesis (11) occurs, thereby disallowing the application of Theorem 1 and its consequential conclusion of existence. Moreover, due to the highly transcendental nature of the elements of the coefficient matrix $A_{3}$, as defined in (41) and inferred from (39), even the determination of an extremal field of the form (37), that solves the Euler-Lagrange equation (21) becomes problematic. In fact, when $k<2 a$, we shall obtain a positive, strictly decreasing sequence $\left\{\hat{\delta}_{n}\right\}_{n \in N}$, of values of the influence parameter $\delta$, corresponding to certain zeros of $\operatorname{det} A_{3}$, such that for each $n, \lim _{\delta \rightarrow \hat{\delta}_{n}}\left|E_{\delta}\left(u_{3, \delta}\right)\right|=\infty$, where $u_{3, \delta}(\cdot) \in \mathscr{A}^{1, p}(\mathscr{B})$ is any extremal field that solves (21). Moreover, when $\delta=\hat{\delta}_{n}$, we show that there does not exist a field $u(\cdot) \in \mathscr{A}^{1, p}(\mathscr{B})$ that satisfies (21). Also, by studying (21) as a Fredholm integral equation of the second kind, we shall identify a second sequence of values of the influence parameter $\delta$, corresponding to other zeros of $\operatorname{det} A_{3}$, which completely characterize the extremal fields $u(\cdot) \in \mathscr{A}^{1, p}(\mathscr{B})$ that do solve $(21)$.

5.1. General extremal fields: Existence and asymptotics. First some remarks concerning coercivity which, according to the coercivity hypothesis (11), is related to the aggregate sign of $k-a_{\delta}(\cdot)$ on the body $\mathscr{B}$. Recalling (43), we see that $k-a_{\delta}(\cdot)$ is bounded below away from zero on $(0, l)$ whenever $2 a-k<2 a e^{\frac{-l}{2 \delta}}$. This is equivalent to the condition that

$$
\delta^{-1} \in\left(0, \frac{-2}{l} \ln \left(1-\frac{k}{2 a}\right)\right)=:\left(0, \frac{1}{\delta^{*}}\right) .
$$

Thus, $\delta^{*}$ is the greatest lower bound to the set of all $\delta>0$ such that the functional $E_{\delta}(\cdot)$ is coercive. It follows that when $\delta \leq \delta^{*}$, the coercivity Hypothesis 3 fails and, as a consequence, the existence theorem, Theorem 1, cannot be applied to such a situation. This, of course, does not mean that a solution of (19) does not exist for $\delta \leq \delta^{*}$, but rather, the loss of coercivity motivates a more delicate and detailed analysis of the existence question. We begin with some preliminary asymptotic limits.

From (39) and (41), we easily find that $\operatorname{det} A_{3}$ has the form

$$
\operatorname{det} A_{3}=8 a^{2}-\left(8 a^{2}+\frac{4 a l}{\delta}(2 a-k)\right) \cos \omega l-\left(4 a l \omega(k-a)-8 a^{2} \delta \omega\right) \sin \omega l .
$$

Of course, the system (41) has a unique solution $\mathbf{c}=A_{3}^{-1} \mathbf{d}$ for $c_{1}, c_{2}$, and $\sigma$ when the above expression is nonzero. To explore this possibility, we note from the definition of $\omega$ in (30) that $\operatorname{det} A_{3}$ has an infinite number of zeros in any neighborhood of $\delta=0$, and that these zeros possess a limit point at $\delta=0$. Further, we note that for $r:=l / \delta$,

$$
\left.\frac{\partial\left(\operatorname{det} A_{3}\right)}{\partial r}\right|_{r=0}=16 a^{2}\left(\frac{k}{a}+4 \frac{a}{k}-4\right)
$$

which is strictly positive for $0<k<2 a$. Since $\left(\operatorname{det} A_{3}\right)_{r=0}=0$, we then may conclude that whenever $0<k<2 a$ there exists a $\bar{\delta}>0$ such that for any $\delta \in$ $(\bar{\delta}, \infty)$, det $A_{3}>0$. Hence, for $\delta$ sufficiently large and finite, (41) is uniquely 
solvable for c. In any case, when $\operatorname{det} A_{3} \neq 0$, we find

$$
\begin{aligned}
c_{1} & =\frac{2 a \Delta \omega(2 a \delta \omega(1+\cos \omega l)+2 a \sin \omega l)}{\operatorname{det} A_{3}}, \\
c_{2} & =\frac{4 a \Delta \omega \sin \left(\frac{l \omega}{2}\right)\left(2 a \delta \omega \cos \left(\frac{\omega l}{2}\right)+2 a \sin \left(\frac{\omega l}{2}\right)\right)}{\operatorname{det} A_{3}}, \\
\sigma & =\frac{2 k(2 a-k) \omega \Delta\left(4 a \delta \omega \cos \omega l-2 a\left(\delta^{2} \omega^{2}-1\right) \sin \omega l\right)}{\operatorname{det} A_{3}} .
\end{aligned}
$$

We denote the resulting extremal strain field of $(37)$ by $e_{3, \delta}(\cdot)$ and its associated displacement field in $\mathscr{A}^{1, p}(\mathscr{B})$ by $u_{3, \delta}(\cdot)$.

In the limiting case $\delta \rightarrow \infty$, we know from (30) that $\omega=O(1 / \delta)$, and from (65) and (66) we obtain

$$
\operatorname{det} A_{3}=\frac{l}{4 \omega \delta}\left(\frac{\partial\left(\operatorname{det} A_{3}\right)}{\partial r}\right)_{r=0} \omega+O\left(\omega^{2}\right) .
$$

This, with (67) and the form for $e_{3, \delta}(\cdot)$ given in (37), then justifies the conclusion that for every $x \in \mathscr{B}$,

$$
e_{3, \infty}(x):=\lim _{\delta \rightarrow \infty} e_{3, \delta}(x)=\frac{\Delta}{l}
$$

While this result follows the same pattern as that observed earlier in the Cases (1) and (2) corresponding to $k \geq 2 a$, when an attempt is made to evaluate the limit of $e_{3, \delta}(\cdot)$ as $\delta \rightarrow 0$, we encounter problems. One complication is due to the large number of zeros for $\operatorname{det} A_{3}$ present in any neighborhood of zero, and another is that $\lim _{\delta \rightarrow 0} \omega=\infty$. These two facts, coupled with (67) and (37) indicate that the extremals of (21) will possess a highly oscillatory spatial behavior and unbounded character with respect to $\delta$ as $\delta$ approaches 0 . This is what we see in Fig. 6.

With a view toward obtaining a complete characterization of the fields $u(\cdot) \in$ $\mathscr{A}^{1, p}(\mathscr{B})$ that solve $(21)$ in the present case when $k<2 a$, we observe that $(21)$ is a Fredholm integral equation of the second kind, which naturally suggests that we first analyze the associated homogeneous problem. For this purpose, let $\phi(\cdot) \in L^{1}(\mathscr{B})$, and define

$$
£_{\delta} \phi(x):=\int_{\mathscr{B}} f_{\delta}(x-z) \phi(z) d z .
$$

Then, for $\sigma=0,(21)$ becomes

$$
e(x)=\frac{1}{k} £_{\delta} e(x),
$$

and from (37) and (39) we see that the general solution of (71) is of the form

$$
e(x)=c_{1} \cos \omega x+c_{2} \sin \omega x
$$

where the pair $\left(c_{1}, c_{2}\right)$ must nontrivially solve the system

$$
\begin{aligned}
c_{1}-\omega \delta c_{2} & =0 \\
(\cos \omega l-\omega \delta \sin \omega l) c_{1}+(\omega \delta \cos \omega l+\sin \omega l) c_{2} & =0 .
\end{aligned}
$$




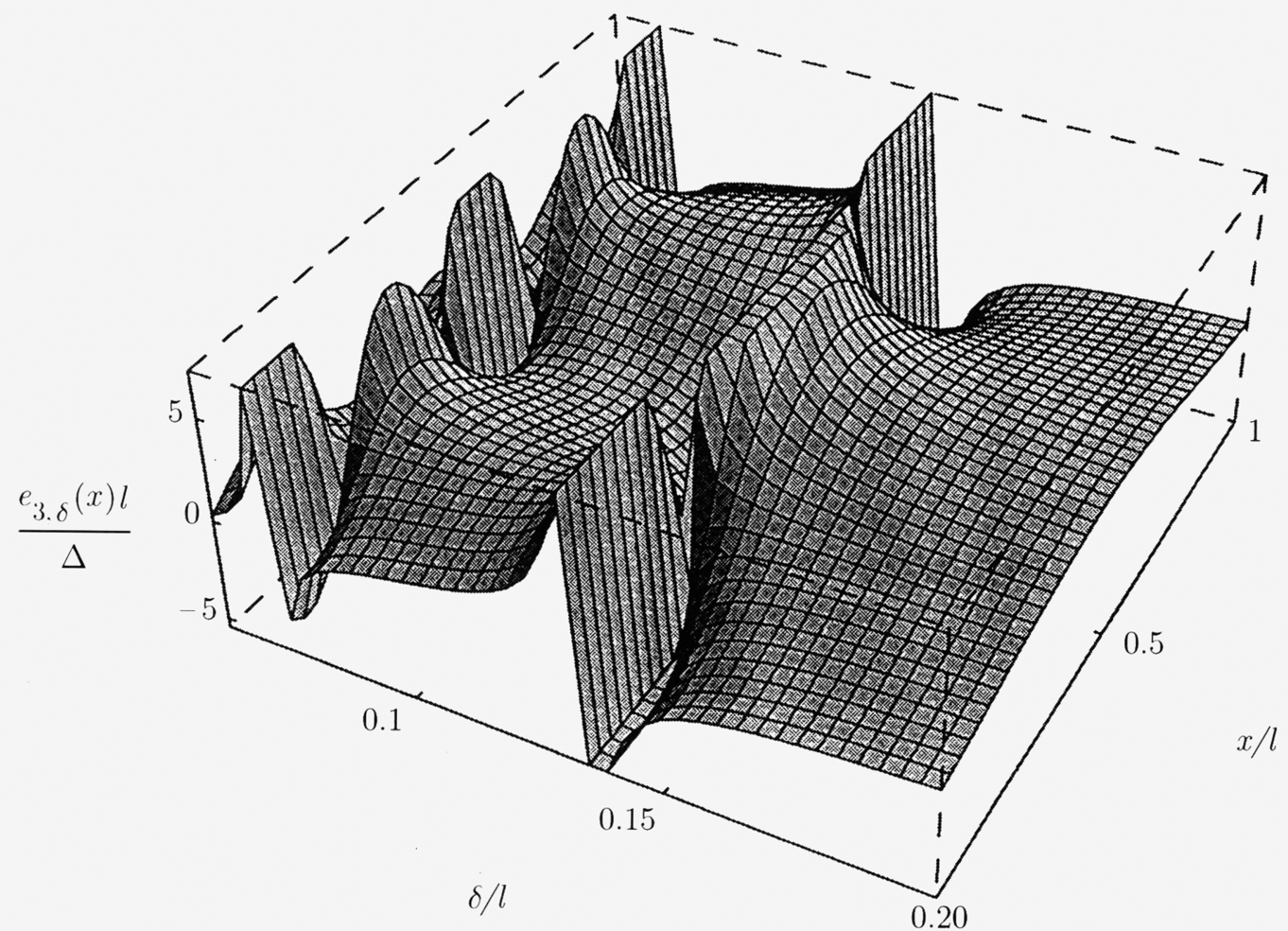

Fig. 6. Solution of the Euler-Lagrange equation (21) and the fixed displacement condition (27) for $k=a$

Clearly, a necessary and sufficient condition that this be the case is that $\operatorname{det} A=0$, where $A$ is the coefficient matrix for (73). Since

$$
\operatorname{det} A=\left(2 \delta \omega \cos \omega l-\left(\delta^{2} \omega^{2}-1\right) \sin \omega l\right),
$$

it follows that $\operatorname{det} A=0$ if and only if

$$
\left\{\begin{array}{ccc}
\omega l=\left(n-\frac{1}{2}\right) \pi & : & k=a ; n \in \mathbb{N} \\
\tan \omega l=\frac{2 \delta \omega}{\delta^{2} \omega^{2}-1} & : & k \neq a
\end{array}\right\} .
$$

Recalling (30), we see that (75) places a restriction on the parameters $k, a, \delta$, and $l$ in order for (71) to be solvable; we say that the ordered set $\Lambda:=\{k, a, \delta, l\} \in \mathbb{R}^{4}$ is an eigenset if its elements combine so as to satisfy $\operatorname{det} A=0$. It will be convenient to separate the possible eigensets into the following two types: $\Lambda$ is a Type I eigenset if

$$
\Lambda \in\left\{\{k, a, \delta, l\}: \sin \omega l=-\frac{k}{a} \delta \omega \text { and } \cos \omega l=-\frac{k}{2 a}\left(\delta^{2} \omega^{2}-1\right)\right\} ;
$$

$\Lambda$ is a Type II eigenset if

$$
\Lambda \in\left\{\{k, a, \delta, l\}: \sin \omega l=\frac{k}{a} \delta \omega \text { and } \cos \omega l=\frac{k}{2 a}\left(\delta^{2} \omega^{2}-1\right)\right\} .
$$

Note that in the case $k=a$, where $\delta \omega=1$ according to (30), this separation corresponds, respectively, to the choice $n=$ even or $n=$ odd in (75). 
In general, then, (71) is solvable if and only if $\Lambda$ is an eigenset. In that case, (73) requires $\operatorname{ker} A=\operatorname{span}\{(\delta \omega, 1)\}$, and so the solution (72) must have the form

$$
e(x)=c_{2}(\delta \omega \cos \omega x+\sin \omega x) \text {. }
$$

Moreover, we see from (76)-(78) that

$$
\begin{aligned}
\int_{\mathscr{B}} e(x) d x & =\frac{c_{2}}{\omega}\{\delta \omega \sin \omega l-\cos \omega l+1\} \\
& =\left\{\begin{array}{ccc}
0 & : & \Lambda \text { is a Type I eigenset } \\
2 \frac{c_{2}}{\omega} & : & \Lambda \text { is a Type II eigenset }
\end{array}\right\} .
\end{aligned}
$$

Let us now require $e(\cdot)$ to satisfy the fixed displacement condition (27). It is clear from (79) that when $\Lambda$ is of Type II, (71) has a unique solution which satisfies (27) for every $\Delta \in \mathbb{R}$ and that solution is identically zero if and only if $\Delta=0$. Explicitly, we have

$$
e_{3, \delta}(x)=\frac{\omega \Delta}{2}(\delta \omega \cos \omega x+\sin \omega x) .
$$

When $\Lambda$ is a Type I eigenset, we conclude from (79) that there is a one-parameter family of solutions of (71), all satisfying the zero fixed displacement condition (i.e., $\Delta=0$ ), of the form given in (78). We note, from (53) and the fact that here we are setting $\sigma=0$, that the total stored energy of the family (78) is zero, independent of $c_{2}$.

Having completely solved the homogeneous problem, (21) with $\sigma=0$, and characterized those fields that satisfy the fixed displacement condition (27), we now use these results to completely analyze the case $\sigma \neq 0$. Thus, we now consider

$$
e(x)=\frac{1}{k} f_{\delta} e(x)+\frac{\sigma}{2 k},
$$

coupled with the associated fixed displacement condition (27). Recalling the expression for $\operatorname{det} A_{3}$ in (65), we see that

$$
\operatorname{det} A_{3}=\left\{\begin{array}{ccc}
0 & : & \Lambda \text { is a Type I eigenset } \\
16 a^{2} & : & \Lambda \text { is a Type II eigenset }
\end{array}\right\} .
$$

Thus, if $\Lambda$ is a Type II eigenset, there is a unique solution of (81) of the form (37), (67), which satisfies the fixed displacement condition (27) for every $\Delta \in \mathbb{R}$; that solution is nonconstant, and is identically zero if and only if $\Delta=0$. In any case, however, by $(67)_{3}$ it follows that $\sigma=0$, and this reduces (81) to the homogeneous case (71). Unfortunately, when $\{k, a, \delta, l\}$ is not an eigenset, the general problem of determining whether or not $\operatorname{det} A_{3}$ is zero is algebraically complicated and not readily solvable. Recalling the general form for $A_{3}$ as implied by (39) and (41), we instead have the following

LEMMA 1. The linear equation

$$
A_{3} \mathbf{c}=\mathbf{d},
$$

as defined in (39) and (41), has a solution $\mathbf{c}=\left(c_{1}, c_{2}, \sigma\right)$ for $\mathbf{d}=(0,0, \Delta) \neq \mathbf{0}$ if and only if either $\Lambda=\{k, a, \delta, l\}$ is a Type I eigenset or $\operatorname{det} A_{3} \neq 0$. 
Proof. Let $\Delta \neq 0$ and suppose that $\mathbf{x}=\left(x_{1}, x_{2}, x_{3}\right) \in \operatorname{ker} A_{3}^{\mathrm{T}}$. Then, $\langle\mathbf{d}, \mathbf{x}\rangle=$ $\Delta x_{3}$ and (83) will have a solution if and only if $x_{3}=0$. Here $\langle\cdot, \cdot\rangle$ is the usual inner product on $\mathbb{R}^{3}$. Thus, with $A_{3}^{\mathrm{T}} \mathbf{x}=\mathbf{0}$ it readily follows that

$$
x_{3}=0 \Leftrightarrow x_{1}+x_{2}=0,
$$

and, if $\mathbf{x} \neq \mathbf{0}$, that

$$
\delta \omega+\sin \omega l+\delta \omega \cos \omega l=0
$$

and

$$
1-\cos \omega l+\delta \omega \sin \omega l=0 .
$$

But, according to (76), this implies that $\Lambda$ is a Type I eigenset. On the other hand, if $\operatorname{ker} A_{3}^{\mathrm{T}}=\{\mathbf{0}\}$, then $\mathbf{x}=\mathbf{0}$, and we must have $\operatorname{det} A_{3} \neq 0$. Thus, we conclude that

$$
\mathbf{d} \perp \operatorname{ker} A_{3}^{\mathrm{T}} \Leftrightarrow\left\{\Lambda \text { is a Type } \mathrm{I} \text { eigenset or } \operatorname{det} A_{3} \neq 0\right\}
$$

to complete the proof.

Since we are interested here in the situation $\sigma \neq 0$, and since $\sigma$ must vanish if $\Lambda$ is a Type II eigenset, then according to this lemma, we need only consider the two possibilities: Either $\operatorname{det} A_{3} \neq 0$ and $\Lambda$ is not an eigenset, in which case the unique solution of (81) and the fixed displacement condition (27) is given by (37) and (67), or $\Lambda$ is a Type I eigenset. For the latter case, we know that $\operatorname{det} A_{3}=0$ and we see from the definition of $A_{3}$ in (39) and (41), and $\omega$ in (30) that

$$
A_{3}=\left(\begin{array}{ccc}
k & -k \delta \omega & \frac{a}{k-2 a} \\
k & -k \delta \omega & \frac{k-2 a}{a} \\
-\frac{k \delta}{a} & \frac{k \delta^{2} \omega}{a} & \frac{l}{2(k-2 a)}
\end{array}\right) .
$$

Performing a standard Gaussian reduction and simplifying we arrive at two results for the case when $\Lambda$ is of Type I. First, by (37), (39), (41), and (88), the general solution of $(81)$ and the fixed displacement condition (27) is given by the one-parameter family

$$
e(x)=c_{2}(\delta \omega \cos \omega x+\sin \omega x)-\frac{2 a \Delta}{k(l+2 \delta)} \cos \omega x+\frac{\Delta}{l+2 \delta} .
$$

Secondly, we find

$$
\sigma=\frac{2(k-2 a) \Delta}{l+2 \delta}
$$

Recalling that $k<2 a$, we see that the stress is of opposite sign to the displacement, which is contrary to physical reasoning and suggests that such an equilibrium state would be unstable. It is of interest to observe from (53) and (90) that when $\Lambda$ is a Type I eigenset, the total stored energy $E_{\delta}(u)$ for an extremal $u(\cdot) \in \mathscr{A}^{1, p}(\mathscr{B})$, as implied by $u^{\prime}(x)=e(x)$ and (89), is nonpositive, finite, and independent of $c_{2}$.

5.2. Stability analysis. In order to better understand the instabilities in the material model for $k<2 a$, we next observe the following elementary properties of Type I eigensets. Suppose that the three parameters $0<k<2 a$ and $l>0$ appearing in the ordered set $\Lambda=\{k, a, \delta, l\}$ are given and fixed. Then, a brief 
inspection of the definition in (75) and (76) implies that there is a strictly decreasing sequence $\wp_{I}:=\left\{\delta_{(n)}\right\}_{n \in N}$ of positive, real numbers such that each collection $\left\{k, a, \delta_{(n)}, l\right\}$ is a Type I eigenset. Moreover, $\wp_{I}$ is bounded above, and $\lim _{n \rightarrow \infty} \delta_{(n)}=0$. Thus, $\delta_{(1)} \in \wp_{I}$ is the largest member of the sequence. Similarly, if instead we hold $k, a$, and $\delta$ fixed in the ordered set $\Lambda$, then there is a strictly increasing sequence $\aleph_{I}:=\left\{l_{(n)}\right\}_{n \in N}$ of positive, real numbers such that each collection $\left\{k, a, \delta, l_{(n)}\right\}$ is also a Type I eigenset. In this case, $\aleph_{I}$ is bounded below by $l_{(1)}$ with $\lim _{n \rightarrow \infty} l_{(n)}=\infty$. Identical observations based upon (75) and (77) also hold for Type II eigensets, whose relevant sequences we denote by $\wp_{I I}:=\left\{\delta^{(n)}\right\}_{n \in N}$ and $\aleph_{I I}:=\left\{l^{(n)}\right\}_{n \in N}$, respectively. It so happens that for every $n \in \mathbb{N}$, we have $\delta_{(n)} \in\left(\delta^{(n+1)}, \delta^{(n)}\right)$ and $l_{(n)} \in\left(l^{(n)}, l^{(n+1)}\right)$, which roughly stated says that "between" any two Type I eigensets there is a Type II eigenset.

It is clear from (82) that $\operatorname{det} A_{3}$ vanishes at Type I eigensets but not at the eigensets of Type II. On the other hand, it follows from (30), (65), and (76) that

$$
\left.\frac{1}{a^{2}} \frac{d\left(\operatorname{det} A_{3}\right)}{d \delta}\right|_{\delta=\delta_{(n)}}=\frac{4(2 a-k)}{\delta_{(n)}^{2}}\left(2+\frac{1}{\delta_{(n)}}\right)>0 .
$$

Thus we see that for each $n \in \mathbb{I N}$ there must be a $\hat{\delta}_{(n)} \in\left(\delta_{(n+1)}, \delta_{(n)}\right)$ such that $\operatorname{det} A_{3}\left(\hat{\delta}_{(n)}\right)=0$. By (53), (67) ${ }_{3}$, and (74) we know that if $u_{3, \delta}(\cdot) \in \mathscr{A}^{1, p}(\mathscr{B})$ is an extremal such that $\operatorname{det} A_{3} \neq 0$, then

$$
E_{\delta}\left(u_{3, \delta}\right)=\frac{2 a k \omega \Delta^{2}(2 a-k) \operatorname{det} A}{\operatorname{det} A_{3}} .
$$

Since $\operatorname{det} A$ is continuous in $\delta$ and $\operatorname{det} A\left(\hat{\delta}_{(n)}\right) \neq 0$, we may conclude from (91) and (92) that for every $n \in \mathbb{N}$,

$$
\lim _{\delta \rightarrow \hat{\delta}_{(n)}} E_{\delta}\left(u_{3, \delta}\right)= \pm \infty
$$

This highly irregular behavior of an extremal field, and our earlier conclusion concerning the boundedness of $E_{\delta}(\cdot)$ on extremals when $\Lambda$ is a Type I eigenset, suggests that there is a $\delta_{0} \in\left(0, \delta_{(1)}\right)$ such that for every $\delta<\delta_{0}$, the functional $E_{\delta}(\cdot)$ will cease to be bounded below in $\mathscr{A}^{1, p}(\mathscr{B})$. If this is the case, then the material will be globally unstable when $\delta<\delta_{0}$. This is the essence of

Theorem 3. Let $0<k<2 a$ and $l>0$ be given. Then, $\exists \delta_{0} \in\left(0, \delta_{(1)}\right)$ such that $\forall \delta \in\left(0, \delta_{0}\right)$,

$$
\inf _{u \in \mathscr{A}^{1, p}(\mathscr{B})} E_{\delta}(u)=-\infty .
$$

Proof. Let $0 \leq x_{1}<x_{2}<x_{3} \leq l$ be given. Define

$$
e_{\lambda}(x):=\left\{\begin{array}{ccc}
\lambda & : & x \in\left[x_{1}, x_{2}\right] \\
\phi(\lambda) & : & x \in\left(x_{2}, x_{3}\right] \\
0 & : & x \in \mathscr{B}-\left[x_{1}, x_{3}\right]
\end{array}\right\},
$$


where $\lambda \in \mathbb{R}$, and $\phi(\lambda)$ is determined so that (95) satisfies the fixed displacement condition (27), i.e.,

$$
\lambda\left(x_{2}-x_{1}\right)+\phi(\lambda)\left(x_{3}-x_{2}\right)=\Delta .
$$

Hence, if we let $u_{\lambda}(\cdot) \in \mathscr{A}^{1, p}(\mathscr{B})$ be such that $u_{\lambda}^{\prime}(\cdot)=e_{\lambda}(\cdot)$, then with the aid of (95) and (6), for the particular model of Sec. 2, we find that

$$
\begin{aligned}
E_{\delta}\left(u_{\lambda}\right)= & (k-2 a) \alpha \lambda^{2}+(k-2 a) \beta[\phi(\lambda)]^{2} \\
& -2 a \delta\left(\lambda^{2}\left(e^{-\alpha / \delta}-1\right)+[\phi(\lambda)]^{2}\left(e^{-\beta / \delta}-1\right)\right)-2 a \delta \lambda \phi(\lambda) P_{\delta},
\end{aligned}
$$

where $\alpha:=x_{2}-x_{1}, \beta:=x_{3}-x_{2}$ and $P_{\delta}:=\left(1-e^{-\alpha / \delta}-e^{-\beta / \delta}+e^{-(\alpha+\beta) / \delta}\right)$. Upon using (96) we see that

$$
\begin{aligned}
E_{\delta}\left(u_{\lambda}\right)= & c(k, a, \alpha, \beta, \delta, \Delta)+\left\{\frac{4 a \delta \alpha \Delta}{\beta^{2}}\left(e^{-\frac{\beta}{\delta}}-1\right)-\frac{2 \alpha(k-2 a) \Delta}{\beta}-\frac{2 a \delta \Delta P_{\delta}}{\beta}\right\} \lambda \\
& +\left\{(k-2 a) \alpha+\frac{(k-2 a)}{\beta} \alpha^{2}-2 a \delta\left(e^{-\alpha / \delta}-1\right)\right. \\
& \left.-\frac{2 a \delta \alpha^{2}}{\beta^{2}}\left(e^{-\beta / \delta}-1\right)+\frac{2 a \alpha \delta P_{\delta}}{\beta}\right\} \lambda^{2}
\end{aligned}
$$

where $c(k, a, \alpha, \beta, \delta, \Delta)$ is a real constant. Now, for simplicity, we assume that $\alpha=\beta=l / 2$. Then, the coefficient of $\lambda^{2}$ in (98) reduces to the form

$$
h(\delta):=a l\left\{\left(\frac{k}{a}-2\right)-\frac{4 \delta}{l}\left(e^{-\frac{1}{2 \delta}}-1\right)+\frac{2 \delta}{l}\left(e^{-\frac{1}{2 \delta}}-1\right)^{2}\right\},
$$

and we see that

$$
\lim _{\delta \rightarrow \infty} h(\delta)=k l>0, \quad \lim _{\delta \rightarrow 0} h(\delta)=\left(\frac{k}{a}-2\right) a l<0,
$$

for every $0<k<2 a$, and that $h^{\prime}(\delta)>0$ for every $\delta \in(0, \infty)$.

In the remainder of this proof, we shall show that there is a single positive root $\delta_{0}$ of $h(\delta)=0$ which is less than $\delta_{(1)}$ and such that for any $\delta \in\left(0, \delta_{0}\right), h(\delta)$ is negative. Then, for any such $\delta$ it will follow from (98) that $E_{\delta}\left(u_{\lambda}\right)$ is unbounded below in the limit as $\lambda \rightarrow \infty$, as claimed in (94). ${ }^{5}$ Specifically, let us consider $h(\delta) /(a l)$ from (99) as a continuous function $g(\cdot, \cdot)$ of the two variables $(k / a, l / \delta) \in \Omega:=(0,2) \times(0, \infty) \subset \mathbb{R}^{2}$. Then, it follows from (100) that the point set $\Omega_{0}:=\{(k / a, l / \delta): h(\delta)=0\}$ consists of a single curve that separates $\Omega$ into two disjoint, connected, open sets, namely $\Omega_{+}:=\{(k / a, l / \delta): g(k / a, l / \delta)>0\}$ and $\Omega_{-}:=\{(k / a, l / \delta): g(k / a, l / \delta)<0\}$, where $\Omega=\Omega_{0} \cup \Omega_{+} \cup \Omega_{-}$. The point

${ }^{5}$ Certainly, for $k$ and $a$ such that $0<k<2 a$ and $\alpha, \beta>0$, one may use (98) to numerically determine the maximum value of $\delta_{0}$ such that (94) holds for a field of the form (95). This computation is easily completed when $k$ and $a$ are given. We performed a number of numerical computations for various choices of the parameters $\alpha$ and $\beta$, all of which indicated that the value of $\delta_{0}$ is maximized when $\alpha=\beta=l / 2$. We have no formal proof of this, however. 


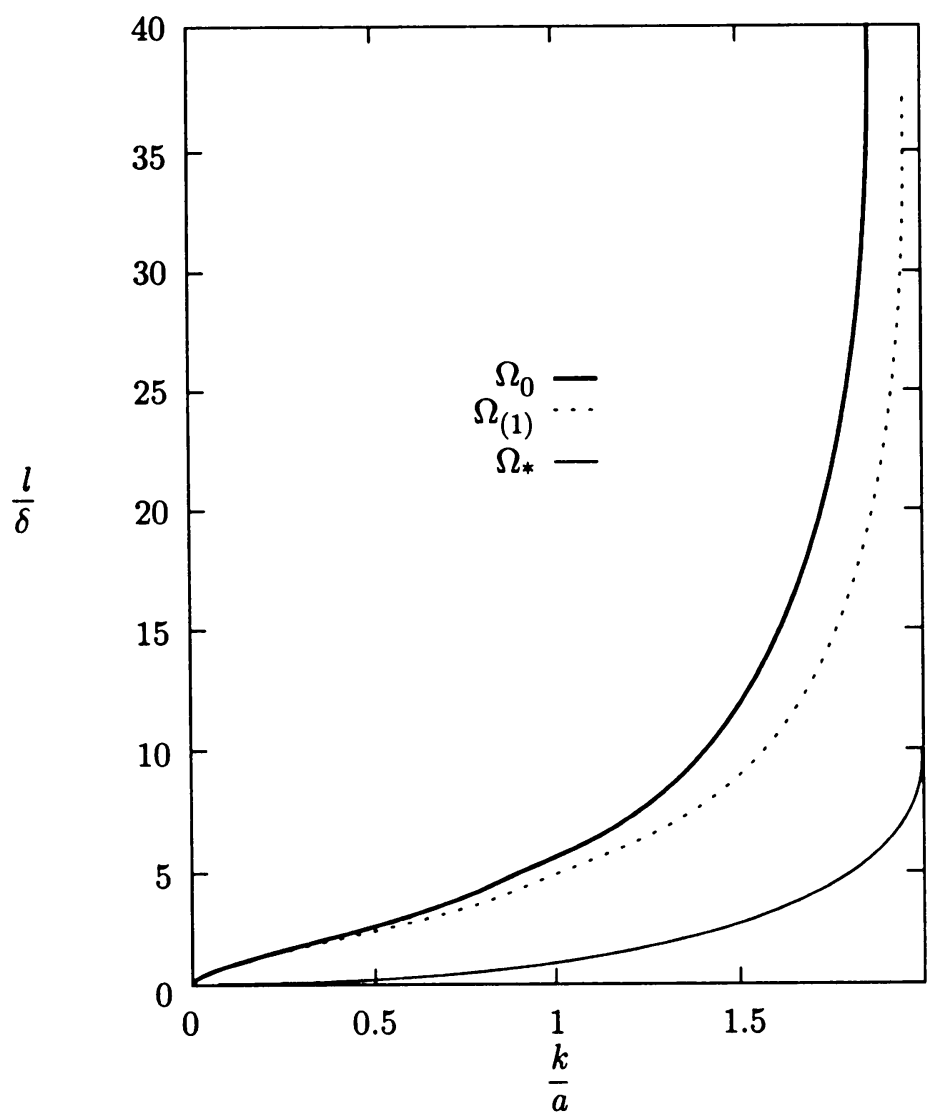

FIG. 7. Admissible values of $l / \delta$ and $k / a$ for (i) the curve $\Omega_{0}$ defined by $h(\delta)=0$ from (99); (ii) the curve $\Omega_{(1)}$ which defines the maximal Type I eigenset $\left\{k, a, \delta_{(1)}, l\right\}$ from (30) and (76); (iii) the curve $\Omega_{*}$ determined by the coercivity condition (64) for $\delta^{*}$.

set $\Omega_{0}$ is determined by a simple numerical computation, and this is shown in Fig. 7. For fixed parameters $0<k<2 a$ and $l>0$ and $\delta \in\left(0, \delta_{0}\right)$, the function $h(\delta)$ is indeed negative. Similarly, we may use (30) to write (76) in terms of the variables $(k / a, l / \delta) \in \Omega$ and obtain the curve $\Omega_{(1)}$ that corresponds to the eigenset $\left\{k, a, \delta_{(1)}, l\right\}$ of Type I which contains the largest member $\delta_{(1)}$ in the decreasing sequence $\wp_{I}$. We also have shown this in Fig. 7, where it is clear that for fixed parameters $0<k<2 a$ and $l>0$ we have $\delta_{0} \in\left(0, \delta_{(1)}\right)$.

Given $k, a$, and $l$ such that $0<k<2 a$ and $l>0$, Theorem 3 provides sufficient conditions on the parameter $\delta>0$ to ensure that the total stored energy functional $E_{\delta}(\cdot)$ is unbounded below on $\mathscr{A}^{1, p}(\mathscr{B})$. Superimposed on Fig. 7 is a graph, which we denote by $\Omega_{*}$, of $l / \delta^{*}$ vs. $k / a$ from the coercivity condition (64). Recall, $\delta^{*}$ is the greatest lower bound to the set of all positive $\delta$ where the functional $E_{\delta}(\cdot)$ is coercive. From this figure we see that for fixed $k, a$, and $l$, as $\delta$ runs through a decreasing sequence, the functional $E_{\delta}(\cdot)$ first loses its coercivity at $\delta=\delta^{*}$ and then, after passing $\delta=\delta_{0}<\delta_{(1)}<\delta^{*}$, it is unbounded below on 
$\mathscr{A}^{1, p}(\mathscr{B})$. In the remainder of this work we shall investigate the sign of the second variation of $E_{\delta}(\cdot)$ in an attempt to determine the stability character of $E_{\delta}(\cdot)$ in the "stability gap" $\left[\delta_{0}, \delta^{*}\right]$, i.e., between $\Omega_{0}$ and $\Omega_{*}$ in Fig. 7. We begin by considering this question for the case when $\Lambda=\{k, a, \delta, l\}$ is a Type I eigenset corresponding to either the maximal $\delta=\delta_{(1)}$ in $\wp_{I}$ or, equivalently, the minimal $l=l_{(1)}$ in $\aleph_{I}$. These values lie on the curve $\Omega_{(1)}$ in Fig. 7 .

First, suppose $u(\cdot) \in \mathscr{A}^{1, p}(\mathscr{B})$ is a solution of the minimization problem (19), and introduce the set of null fields

$$
\mathscr{A}_{0}^{1, p}(\mathscr{B}):=\left\{\eta(\cdot) \in \mathscr{A}^{1, p}(\mathscr{B}): \Delta=0\right\} .
$$

Then, for every $\epsilon>0$ and null field $\eta(\cdot)$, we see that $u_{\epsilon}(\cdot):=u(\cdot)+\epsilon \eta(\cdot) \epsilon$ $\mathscr{A}^{1, p}(\mathscr{B})$. Necessary conditions for $u(\cdot)$ to solve $(19)$ are

$$
\left.\frac{\partial E_{\delta}\left(u_{\epsilon}\right)}{\partial \epsilon}\right|_{\epsilon=0}=0 ;\left.\quad \frac{\partial^{2} E_{\delta}\left(u_{\epsilon}\right)}{\partial \epsilon^{2}}\right|_{\epsilon=0} \geq 0 .
$$

The first condition in (102) reduces essentially to the statement of Theorem 1 and the necessity of the Euler-Lagrange equation (21). Given $\eta(\cdot) \in \mathscr{A}_{0}^{1, p}(\mathscr{B})$, we observe that the second condition in (102) reduces to

$$
\delta^{2} E_{\delta}(u ; \eta):=\left.\frac{\partial^{2} E_{\delta}\left(u_{\epsilon}\right)}{\partial \epsilon^{2}}\right|_{\epsilon=0}=2 \int_{\mathscr{B}}\left\{k \eta^{\prime 2}(x)-\eta^{\prime}(x) £_{\delta} \eta^{\prime}(x)\right\} d x \geq 0,
$$

where the expression $\delta^{2} E_{\delta}(u ; \eta)$ denotes the second variation of the functional $E_{\delta}(\cdot)$ at $u(\cdot)$ in the direction $\eta(\cdot)$. We say that a solution $u(\cdot) \in \mathscr{A}^{1, p}(\mathscr{B})$ of the Euler-Lagrange equation (21) is locally stable if for every $\eta(\cdot) \in \mathscr{A}_{0}^{1, p}(\mathscr{B})$ we have $\delta^{2} E_{\delta}(u ; \eta)>0$. If instead $\delta^{2} E_{\delta}(u ; \eta)<0$, then we say that the given displacement field is unstable. We emphasize here that the positivity of the second variation of $E_{\delta}(\cdot)$ at a field $u(\cdot)$ does not necessarily guarantee that the field $u(\cdot)$ is globally stable in the sense that it is a solution of the complete minimization problem (19).

To determine those $\delta>0$ for which there is a null field $\eta(\cdot)$ with the property that $\delta^{2} E_{\delta}(u ; \eta)<0$, we minimize the second variation (103) over the class of null functions $\mathscr{A}_{0}^{1, p}(\mathscr{B})$. Since $\delta^{2} E_{\delta}(u ; \eta)$ is a positive scalar multiple of the original total stored energy $E_{\delta}(\cdot)$, we have, by Theorem 2 , that if $\eta(\cdot) \in \mathscr{A}_{0}^{1, p}(\mathscr{B})$ is an extremal of the second variation, then there exists a $\mu \in \mathbb{R}$ such that

$$
\eta^{\prime}(x)=\frac{1}{k} \int_{\mathscr{B}} f_{\delta}(x-z) \eta^{\prime}(z) d z+\frac{\mu}{2 k} .
$$

Now suppose $\Lambda=\{k, a, \delta, l\}$ is a Type I eigenset. Then, by our previous discussion concerning (71)-(80) we know that there is a nontrivial null function $\eta(\cdot) \in \mathscr{A}_{0}^{1, p}(\mathscr{B})$ such that $\eta(\cdot)$ solves (104) when $\mu=0$. It is then immediate from (103) that in this case $\delta^{2} E_{\delta}(u ; \eta)=0$. This extremal function $\eta(\cdot)$ can now be used as the building block for a classical "Jacobi-type" stability argument. Recalling the sequences $\wp_{I}=\left\{\delta_{(n)}\right\}_{n \in N}$ and $\aleph_{I}=\left\{l_{(n)}\right\}_{n \in N}$ corresponding to Type I eigensets, we have the following theorem. 
TheOREM 4 (JACOBI). Let $0<k<2 a$ and $\delta>0$ be given. Let $l_{(1)}>0$ be the minimal element of $\aleph_{I}$, so that $\left\{k, a, \delta, l_{(1)}\right\}$ is a Type I eigenset, and let $l>l_{(1)}$. Suppose that $E_{\delta}(\cdot)$ denotes the total stored energy functional for the body $\mathscr{B}:=(0, l)$, and that $u(\cdot) \in \mathscr{A}^{1, p}(\mathscr{B})$ satisfies the Euler-Lagrange equation (21). Then, $\exists \eta(\cdot) \in \mathscr{A}_{0}^{1, p}(\mathscr{B})$ such that

$$
\delta^{2} E_{\delta}(u ; \eta)<0 .
$$

Proof. From the previous discussion concerning (71), there is an $\eta(\cdot) \in \mathscr{A}_{0}^{1, p}\left(\mathscr{B}_{(1)}\right)$ such that

$$
\eta^{\prime}(x)=\frac{1}{k} \int_{\mathscr{B}_{(1)}} f_{\delta}(x-z) \eta^{\prime}(z) d z
$$

for every $x \in \mathscr{B}_{(1)}$, where $\mathscr{B}_{(1)}:=\left(0, l_{(1)}\right) \subset \mathscr{B}$. Let $\hat{\eta}(\cdot) \in \mathscr{A}_{0}^{1, p}(\mathscr{B})$ be defined such that

$$
\hat{\eta}^{\prime}(x):=\left\{\begin{array}{ccc}
\eta^{\prime}(x) & : & x \in\left(0, l_{(1)}\right) \\
0 & : & x \in\left[l_{(1)}, l\right)
\end{array}\right\} .
$$

Then, using (103), (106), and (107) we see that

$$
\delta^{2} E_{\delta}(u ; \hat{\eta})=2 \int_{\mathscr{B}_{(1)}}\left\{k \eta^{\prime 2}(x)-\eta^{\prime}(x) \int_{\mathscr{B}_{(1)}} f_{\delta}(x-z) \eta^{\prime}(z) d z\right\} d x=0 .
$$

While (108) shows that $\delta^{2} E_{\delta}(u ; \cdot)$ evaluated at $\hat{\eta}(\cdot) \in \mathscr{A}_{0}^{1, p}(\mathscr{B})$ is zero, we will now show that $\delta^{2} E_{\delta}(u ; \cdot)$ is not minimized at $\hat{\eta}(\cdot)$ over the set $\mathscr{A}_{0}^{1, p}(\mathscr{B})$. This will establish (105).

To carry this out, we suppose, for contradiction, that $\hat{\eta}(\cdot)$ is a minimizer of $\delta^{2} E_{\delta}(u ; \cdot)$ over $\mathscr{A}_{0}^{1, p}(\mathscr{B})$. Then, the first variation condition (104) requires $\hat{\eta}(\cdot)$ to satisfy

$$
\hat{\eta}^{\prime}(x)=\frac{1}{k} \int_{\mathscr{B}} f_{\delta}(x-z) \hat{\eta}^{\prime}(z) d z+\rho
$$

for all $x \in \mathscr{B}$ and for some real constant $\rho$. Because of (107), this yields the following necessary conditions:

$$
\eta^{\prime}(x)=\frac{1}{k} \int_{\mathscr{B}_{(1)}} f_{\delta}(x-z) \eta^{\prime}(z) d z+\rho \quad \forall x \in \mathscr{B}_{(1)}
$$

and

$$
\int_{\mathscr{B}_{(1)}} f_{\delta}(x-z) \eta^{\prime}(z) d z+\rho=0 \quad \forall x \in\left[l_{(1)}, l\right) .
$$

Using (106), we then see that $\rho=0$, so that if $\hat{\eta}(\cdot)$ is to be a minimizing field, then (111) must hold with $\rho=0$. But, by analogy between (71) and (106), and the general solution (78) of (71), we know that $\eta^{\prime}(\cdot)$ must be of the form $\eta^{\prime}(x)=$ $c(\delta \omega \cos \omega x+\sin \omega x)$, where $c$ is an arbitrary constant. Thus, carrying out the relevant integration using (18), we find that

$$
\int_{\mathscr{B}_{(1)}} f_{\delta}(x-z) \eta^{\prime}(z) d z=-k \delta \omega c e^{-\left(x-l_{1}\right) / \delta} \neq 0 .
$$


Since this contradicts (111) for $\rho=0$, we conclude that $\hat{\eta}(\cdot)$ does not satisfy the first variation condition (109), and therefore it cannot minimize $\delta^{2} E_{\delta}(u ; \cdot)$ over $\mathscr{A}_{0}^{1, p}(\mathscr{B})$. In light of $(108)$, the proof is completed.

In physical terms, Theorem 4 says that when all other parameters are fixed with $k<2 a$, if the length $l$ of $\mathscr{B}$ is large enough $\left(>l_{(1)}\right)$ then an extremal of $E_{\delta}(\cdot)$ in $\mathscr{A}^{1, p}(\mathscr{B})$ is unstable. Alternatively, if all other parameters except the influence parameter $\delta$ are fixed, as above, then such an extremal is unstable if $\delta$ is small enough $\left(<\delta_{(1)}\right)$. This is the content of

Corollary 3. Let $0<k<2 a$ and $l>0$ be given. Let $\delta_{(1)}>0$ be the maximal element of the sequence $\wp_{I}$, so that $\left\{k, a, \delta_{(1)}, l\right\}$ is a Type I eigenset, and let $0<\delta<\delta_{(1)}$. Suppose that $u(\cdot) \in \mathscr{A}^{1, p}(\mathscr{B})$ satisfies the Euler-Lagrange equation (21) for $E_{\delta}(\cdot)$. Then, $\exists \eta(\cdot) \in \mathscr{A}_{0}^{1, p}(\mathscr{B})$ such that

$$
\delta^{2} E_{\delta}(u ; \eta)<0 .
$$

Proof. Let $\gamma \in(0,1)$ be such that $\delta=\gamma \delta_{(1)}$ and observe from (21) that $u^{\prime}(\cdot)=$ $e(\cdot)$ is supposed to satisfy

$$
e(x)=\frac{1}{k} \int_{\mathscr{B}} f_{\delta}(x-z) e(z) d z+\alpha \quad \forall x \in \mathscr{B},
$$

for some $\alpha \in \mathbb{R}$. Let $\bar{x}:=x / \gamma$ and $\bar{z}:=z / \gamma$ and define $\hat{e}(\bar{x}):=e(\gamma \bar{x})$. Then, recalling (18), we may rewrite (114) in the form

$$
\hat{e}(\bar{x})=\frac{1}{k} \int_{\mathscr{B}_{(1)}} f_{\delta_{(1)}}(\bar{x}-\bar{z}) \hat{e}(\bar{z}) d \bar{z}+\alpha
$$

for every $\bar{x} \in(0, \bar{l})$, where $\bar{l}:=l / \gamma$ and $\mathscr{B}_{(\bar{l})}:=(0, \bar{l}) \supset \mathscr{B}$. Thus, by applying Theorem 2 we see that $\hat{e}(\cdot)$ satisfies the Euler-Lagrange equation for the functional

$$
\bar{E}_{\delta_{(1)}}(u):=\int_{\mathscr{B}_{(1)}}\left\{k e^{2}(x)-e(x) \int_{\mathscr{B}_{(1)}} f_{\delta_{(1)}}(x-z) e(z) d z\right\} d x
$$

defined for all $u(\cdot) \in \mathscr{A}^{1, p}\left(\mathscr{B}_{(\bar{l})}\right)$. Since $\left\{k, a, \delta_{(1)}, l\right\}$ is a Type I eigenset and, in the present case, $\mathscr{B} \subset \mathscr{B}_{(\bar{l})}$ for $\gamma \in(0,1)$, we may conclude using Theorem 4 that there is an $\eta(\cdot) \in \mathscr{A}_{0}^{1, p}\left(\mathscr{B}_{(\bar{l})}\right)$ such that

$$
\delta^{2} \bar{E}_{\delta_{(1)}}(\hat{u} ; \eta)<0,
$$

where $\hat{u}(\cdot) \in \mathscr{A}^{1, p}\left(\mathscr{B}_{(\bar{l})}\right)$, with $\hat{u}^{\prime}(\cdot)=\hat{e}(\cdot)$, satisfies the Euler-Lagrange equation (115). Finally, by recalling (18) and making a standard change of variables of integration in the expression

$$
\delta^{2} \bar{E}_{\delta_{(1)}}(\hat{u} ; \eta)=2 \int_{\mathscr{B}_{(1)}}\left\{k \eta^{\prime 2}(x)-\eta^{\prime}(x) \int_{\mathscr{B}_{(1)}} f_{\delta_{(1)}}(x-z) \eta^{\prime}(z) d z\right\} d x,
$$


we observe that

$$
\begin{aligned}
\delta^{2} \bar{E}_{\delta_{(1)}}(\hat{u} ; \eta) & =\frac{2}{\gamma} \int_{\mathscr{B}}\left\{k \check{\eta}^{\prime 2}(x)-\check{\eta}^{\prime}(x) \int_{\mathscr{B}} f_{\delta}(x-z) \check{\eta}^{\prime}(z) d z\right\} d x, \\
& =\frac{1}{\gamma} \delta^{2} E_{\delta}(u ; \check{\eta}),
\end{aligned}
$$

where $\check{\eta}^{\prime}(x):=\eta^{\prime}(x / \gamma)$ for every $x \in \mathscr{B}$. Then, since it is clear that $\check{\eta}(\cdot) \in$ $\mathscr{A}_{0}{ }^{1, p}(\mathscr{B})$, we see from $(117),(119)$ and the fact that $\gamma>0$, that (113) holds.

The previous theorem and corollary prove that if $k, a$, and $l$ are given such that $0<k<2 a$ and $l>0$, and if $0<\delta<\delta_{(1)}$, where $\delta_{(1)}$ is uniquely determined from (75) and (76) as the largest element of $\wp_{I}$, then the extremal strain fields derived in Sec. 3 and given by (37) cannot correspond to local minima of $E_{\delta}(\cdot)$ over $\mathscr{A}^{1, p}(\mathscr{B})$. Similarly, if the material parameters $k, a$ and $\delta>0$ are given such that $0<k<2 a$ and $\delta>0$, then the model becomes unstable if the reference length $l$ of the body $\mathscr{B}$ is chosen sufficiently large $\left(l>l_{(1)}\right)$. Given such suitable arbitrary values of $k, a$, and $l$, we observe from Fig. 7 that $\delta_{(1)}$ is strictly less than $\delta^{*}$. Thus, as $\delta$ runs through a decreasing sequence of values, the functional $E_{\delta}(\cdot)$ first loses coercivity at $\delta^{*}$ and then for all $\delta<\delta_{(1)}$ we now know, according to Corollary 3 , that the model is unstable. The value of $\delta=\delta_{0}$, corresponding to the curve $\Omega_{0}$ in Fig. 7 where the model shows global instability, is less than $\delta_{(1)}$ and the ordering $\delta_{0}<\delta_{(1)}<\delta^{*}$ is natural. The "stability gap" referred to above in which the stability character of $E_{\delta}(\cdot)$ is unknown has now been narrowed to those $\delta$ in the interval $\left[\delta_{(1)}, \delta^{*}\right]$, i.e., between $\Omega_{(1)}$ and $\Omega_{*}$ in Fig. 7. In fact, we will now narrow this gap further and show that the maximal member $\delta^{(1)}$ of the sequence $\wp_{I I}$ corresponding to Type II eigensets $\left\{k, a, \delta^{(n)}, l\right\}$ provides a lower bound such that for all $\delta \geq \delta^{(1)}$ the second variation $\delta^{2} E_{\delta}(u ; \cdot)$ at the solution $u(\cdot) \in \mathscr{A}^{1 \cdot p}(\mathscr{B})$ of the Euler-Lagrange equation $(21)$ is positive. Moreover, we will see that $\delta^{(1)}$ is below $\delta^{*}$ and above $\delta_{(1)}$ so that the remaining "stability gap" is again narrowed to those $\delta$ that belong to the interval $\left[\delta_{(1)}, \delta^{(1)}\right)$.

We observed earlier, following (104), that the second variation $\delta^{2} E_{\delta}(u ; \cdot)$ vanishes in a particular direction at $\delta=\delta_{(1)}$, and that there is a direction in which it is negative for every $\delta<\delta_{(1)}$. Based upon an approach discussed in a remark at the end of this paper, it is possible that the "stability gap" can be further narrowed and, in fact, possibly eliminated. For arbitrary $k, a$, and $l$ such that $0<k<2 a$ and $l>0$, we show that there is a $\bar{\delta} \in\left(\delta_{(1)}, \delta^{(1)}\right)$ such that the second variation is strictly positive on $\mathscr{A}_{0}^{1, p}(\mathscr{B})$ for every $\delta \in(\bar{\delta}, \infty)$, thereby narrowing the "stability gap" to $\left[\delta_{(1)}, \bar{\delta}\right] \subset\left[\delta_{(1)}, \delta^{(1)}\right]$. To proceed, we examine the eigenvalue problem for the homogeneous problem (21) (i.e., $\sigma=0$ ), with a goal toward using the equivalency between the eigenvalue problem and its associated minimization problem to conclude the positivity of the second variation $\delta^{2} E_{\delta}(u ; \cdot)$ on an extremal field $u(\cdot) \in \mathscr{A}^{1 \cdot p}(\mathscr{B})$. 
Consider, for $\lambda \in \mathbb{R}$ and $\phi(\cdot) \in L^{2}(\mathscr{B})$,

$$
\phi(x)=\frac{\lambda}{k} £_{\delta} \phi(x)
$$

for all $x \in \mathscr{B}$. By analogy with the Euler-Lagrange equation (21) in Sec. 2, we conclude that a necessary condition for $\phi(\cdot)$ to solve $(120)$ is that $\phi(\cdot)$ satisfy

$$
\phi^{\prime \prime}(x)+\frac{1}{k \delta^{2}}(2 a \lambda-k) \phi(x)=0 \quad \forall x \in \mathscr{B} .
$$

The general solutions of (121) contain two arbitrary, real constants $c_{1}$ and $c_{2}$, and separate into three classes depending upon the sign of $2 a \lambda-k$. Let $C_{i}$ denote the upper left $2 \times 2$ submatrix of $A_{i}$, as inferred from Eqs. (33), (36), and (39), and defined in (41), except that everywhere in $A_{i}$ we replace $a$ by $a \lambda$. Then, by the discussion in Sec. 3, it is clear that solutions of (121) solve (120) if and only if the coefficient matrix $C_{i}$ has a nontrivial kernel, where here the subscript $i=1,2,3$ refers to the cases $2 a \lambda<k, 2 a \lambda=k$, and $2 a \lambda>k$, respectively. We easily see from the linear systems (33) and (36) that if $2 a \lambda \leq k$, then $\operatorname{ker} C_{1}=\operatorname{ker} C_{2}=\{0\}$. Therefore, a solution of (120) is possible only if $2 a \lambda>k$. Thus, by introducing $\kappa>0$ through

$$
\kappa^{2}:=\frac{1}{\delta^{2}}\left(\frac{2 a \lambda}{k}-1\right),
$$

we recall from (73) and (74) that $\operatorname{ker} C_{3} \neq\{0\}$ if and only if

$$
2 \delta \kappa \cos \kappa l=\left(\delta^{2} \kappa^{2}-1\right) \sin \kappa l \text {. }
$$

In this case, similar to our analysis in the first half of this section, we infer that there is a strictly increasing sequence of eigenvalues $\Im:=\left\{\lambda_{n}\right\}_{n \in N}$, having a minimum element, denoted by $\lambda_{1}$, and satisfying $\lim _{n \rightarrow \infty} \lambda_{n}=\infty$. Moreover, we may separate the members of $\Im$ into two distinct classes depending upon whether $n$ is odd

$$
\left\{\sin \kappa l=\frac{k}{a \lambda} \kappa \delta ; \quad \cos \kappa l=\frac{k}{2 a \lambda}\left(\kappa^{2} \delta^{2}-1\right)\right\},
$$

or whether $n$ is even

$$
\left\{\sin \kappa l=-\frac{k}{a \lambda} \kappa \delta ; \quad \cos \kappa l=-\frac{k}{2 a \lambda}\left(\kappa^{2} \delta^{2}-1\right)\right\} .
$$

The eigenfunctions for (120), which we denote by $\phi_{n}(\cdot)$, are therefore of the form

$$
\phi_{n}(x):=c_{n}\left(\kappa_{n} \delta \cos \kappa_{n} x+\sin \kappa_{n} x\right)
$$

where $\kappa_{n}$ has the obvious definition from (122). Also, we conclude from (122), (124), (125), and (126) that

$$
\int_{\mathscr{B}} \phi_{n}(x) d x=\left\{\begin{array}{cll}
0 & : & n \text { is even } \\
2 \frac{c_{n}}{\kappa_{n}} & : & n \text { is odd }
\end{array}\right\} .
$$

Since $2 a \lambda_{n}>k>0$ for every $n \in \mathbb{N}$, it follows that $\Im \subset(0, \infty)$, and therefore, the linear Hilbert-Schmidt operator $f_{\delta}(\cdot)$ is symmetric and positive definite [4]. We 
may assume without loss of generality that for every $n \in \mathbb{N}$, the eigenfunctions $\phi_{n}(\cdot)$ have been normalized so that $\left\|\phi_{n}\right\|_{2}=1$, and in this case we have $c_{n}^{2}=$ $\left(l a \lambda_{n} / k+\delta\right)^{-1}$. Consequently, since the eigenvalues $\lambda_{n}$ are distinct, we have that $\left\langle\phi_{i}, \phi_{j}\right\rangle=\delta_{i j}$, where $\delta_{i j}$ is the usual Kronecker delta, and $\langle\cdot, \cdot\rangle$ denotes the usual inner product on $L^{2}(\mathscr{B})$ [4]. Then, if we define

$$
\mathscr{D}_{n}:=\left\{p(\cdot) \in L^{2}(\mathscr{B}):\left\langle p, \phi_{k}\right\rangle=0 \text { for } k=1, \ldots, n-1\right\},
$$

it follows from the extremal properties of eigenvalues for operators of this type [4, 11] that for every $n \in \mathbb{N}$ and nontrivial $p(\cdot) \in \mathscr{D}_{n}$,

$$
0<\left\langle\varepsilon_{\delta} p, p\right\rangle \leq \frac{k}{\lambda_{n}}\|p\|_{2}^{2},
$$

where equality holds if and only if $p(\cdot) \propto \phi_{n}(\cdot)$. Now, we recall the sequences $\wp_{I}$ and $\wp_{I I}$ which characterized, respectively, the Type I and Type II eigensets $\left\{k, a, \delta_{(n)}, l\right\}$ as defined in (30), (75), (76), and (77) for $n \in \mathbb{N}$. Then, according to (122) and (123) the sequence of eigenvalues $\Im$ possesses the following elementary properties relative to $\wp_{I}$ and $\wp_{I I}$, which we state without proof.

Lemma 2. Let $0<k<2 a$ and $l>0$ be given, and choose $\delta_{(m)} \in \wp_{I}$ and $\delta^{(n)} \in \wp_{I I}$. Then,

(i) if $n \in \mathbb{I N}$ is fixed, $\lambda_{n}$ strictly increases as a function of $\delta$,

(ii) $\delta=\delta_{(m)} \Leftrightarrow \lambda_{2 m}=1$,

(iii) $\delta=\delta^{(n)} \Leftrightarrow \lambda_{2 n-1}=1$.

Then, using (129), we have the following

Theorem 5. Let $0<k<2 a$ and $l>0$ be given, and suppose that $u(\cdot) \in \mathscr{A}^{1, p}(\mathscr{B})$ is a solution of the Euler-Lagrange equation (21) for $\delta \geq \delta^{(1)}$. Then, for every $\eta(\cdot) \in \mathscr{A}_{0}^{1, p}(\mathscr{B})$,

$$
\delta^{2} E_{\delta}(u ; \eta)>0 .
$$

Proof. Suppose $\delta \geq \delta^{(1)}$, choose $\eta(\cdot) \in \mathscr{A}_{0}^{1, p}(\mathscr{B})$, and assume without loss of generality that $\left\|\eta^{\prime}\right\|_{2}=1$. Then, by (103), (128), and (129), we observe that

$$
\begin{aligned}
\delta^{2} E_{\delta}(u ; \eta) & =2 \int_{\mathscr{B}}\left\{k \eta^{\prime 2}(x)-\eta^{\prime}(x) \mathfrak{E}_{\delta} \eta^{\prime}(x)\right\} d x \\
& \geq 2 \int_{\mathscr{B}}\left\{k \eta^{\prime 2}(x)-\eta^{\prime}(x) \frac{k}{\lambda_{1}} \eta^{\prime}(x)\right\} d x \\
& =2 k\left(1-\frac{1}{\lambda_{1}}\right) .
\end{aligned}
$$

By Lemma 2, we may then conclude that $\delta^{2} E_{\delta}(u ; \eta) \geq 0$. However, by (129), we see that the inequality in (131) is strict unless $\eta^{\prime}(\cdot)=\phi_{1}(\cdot)$, and this cannot happen since $\eta(\cdot) \in \mathscr{A}_{0}^{1, p}(\mathscr{B})$ and $\phi_{1}(\cdot)$ satisfies $(127)_{2}$. 


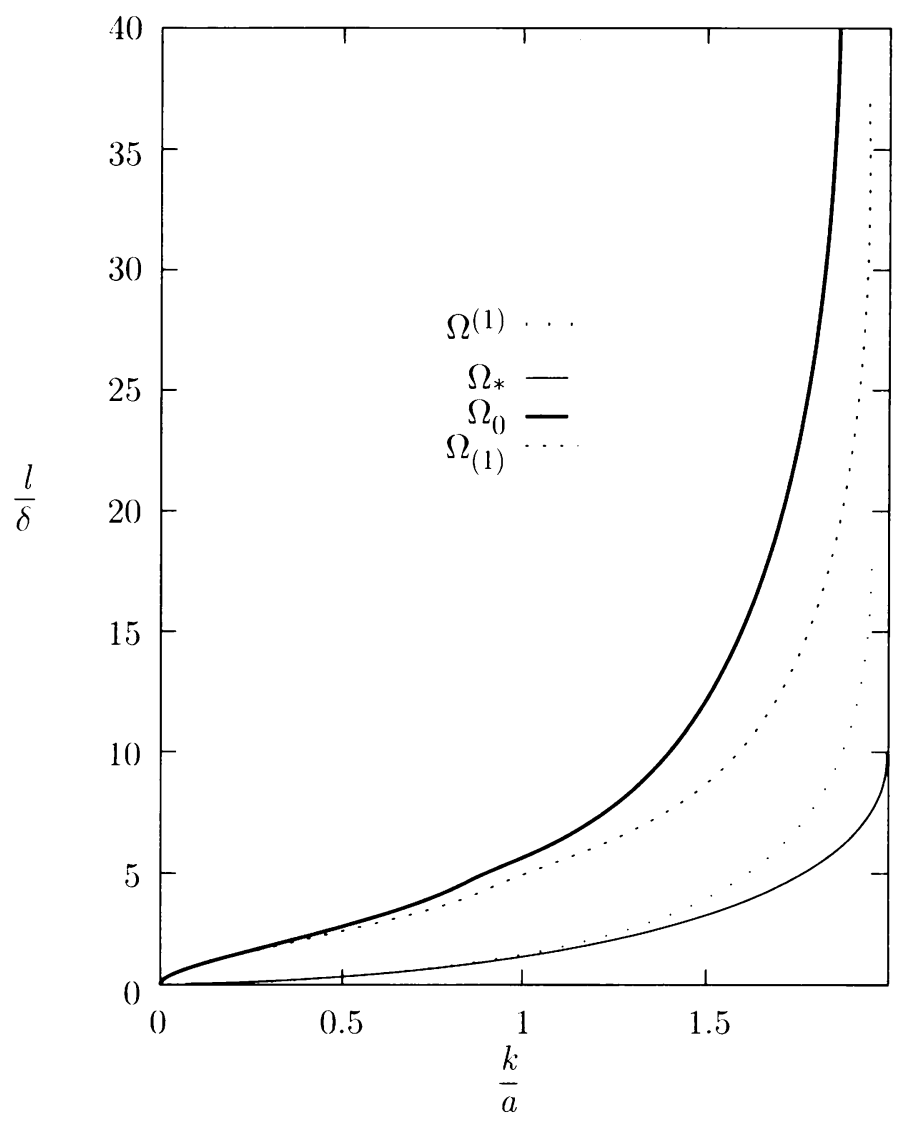

FIG. 8. Stability diagram showing loss of coercivity $\Omega_{*}$, the stability gap bounded by the dotted curves $\Omega_{(1)}$ and $\Omega^{(1)}$, the loss of local stability $\Omega_{(1)}$, and the loss of global stability $\Omega_{0}$.

In Fig. 8 we show all of the information contained in Fig. 7 and in addition we illustrate a graph, denoted by $\Omega^{(1)}$, of $l / \delta^{(1)}$ vs. $k / a$ for the Type II eigenset which corresponds to the maximal value of $\delta=\delta^{(1)}$. Earlier, we observed that $\delta_{(1)}<\delta^{(1)}$ and this is certainly confirmed in Fig. 8. More insightful, however, is the observation that $\delta^{(1)}<\delta^{*}$, where, recall, $\delta^{*}$ defines the coercivity limit in the sense that $E_{\delta}(\cdot)$ is coercive only if $\delta>\delta^{*}$. Because of Theorem 5 , this means that for $k, a$, and $l$ given such that $0<k<2 a$ and $l>0$, and for any $\delta \geq \delta^{(1)}$ the solution $u(\cdot) \in \mathscr{A}^{1, p}(\mathscr{B})$ of the Euler-Lagrange equation (21) is locally stable, even though for $\delta \in\left[\delta^{(1)}, \delta^{*}\right]$ the existence theorem, Theorem 1, for the minimum problem (19) does not apply. With this result, we have narrowed the "stability gap" to those $\delta$ in the interval $\left[\delta_{(1)}, \delta^{(1)}\right)$, as is shown in Fig. 8. In this gap we have not determined the stability character of $E_{\delta}(\cdot)$.

REMARK IN CLOSING. A possible approach to the remaining problem of characterizing the "stability gap" is to use the eigenfunction theory developed above in an attempt to either increase from $\delta_{(1)}$ the threshold for local instability, or to decrease 
from $\delta^{(1)}$ the threshold for local stability. In particular, suppose $u(\cdot) \in \mathscr{A}^{1, p}(\mathscr{B})$ is a solution of the Euler-Lagrange equation (21), and define null functions $\eta(\cdot) \in$ $\mathscr{A}_{0}^{1, p}(\mathscr{B})$ by

$$
\eta(x):=\int_{0}^{x}\left\{b_{1} \phi_{1}(\xi)+w(\xi)\right\} d \xi
$$

where $b_{1} \in \mathbb{R}, \phi_{1}(\cdot)$ is the eigenfunction associated with $\lambda_{1}$, and $w(\cdot) \in \mathscr{D}_{1}$. Then, the definition of $\mathscr{A}_{0}^{1, p}(\mathscr{B})$ coupled with (122) and (127) implies that $w(\cdot)$ has nonzero mass, and that

$$
b_{1}=-\frac{1}{2} \kappa_{1}\left(\frac{l a \lambda_{1}}{k}+\delta\right) \int_{\mathscr{B}} w(x) d x
$$

holds. By substituting (132) and (133) into the second variation (103), and recalling (120) and (129), we observe that

$$
\begin{aligned}
\delta^{2} E_{\delta}(u ; \eta) & =\sigma_{\lambda_{1}}\left(\int_{\mathscr{B}} w(x) d x\right)^{2}+k\|w\|_{2}^{2}-\left\langle\mathscr{L}_{\delta} w, w\right\rangle, \\
& \geq \sigma_{\lambda_{1}}\left(\int_{\mathscr{B}} w(x) d x\right)^{2}+k\left(1-\frac{1}{\lambda_{2}}\right)\|w\|_{2}^{2},
\end{aligned}
$$

where

$$
\sigma_{\lambda_{1}}:=\frac{k}{4}\left(1-\frac{1}{\lambda_{1}}\right)\left(\frac{l a \lambda_{1}}{k}+\delta\right)\left(\frac{2 a \lambda_{1}}{k}-1\right) .
$$

It then follows from Lemma 2 that, given $k, a$, and $l$ such that $0<k<2 a$ and $l>0$, the expression $\sigma_{\lambda_{1}}$ given by (136) is nonpositive for all $\delta \in\left[\delta_{(1)}, \delta^{(1)}\right]$. In this case, we conclude from (135) and the Cauchy-Schwarz inequality that

$$
\delta^{2} E_{\delta}(u ; \eta) \geq\left(\sigma_{\lambda_{1}} l+k\left(1-\frac{1}{\lambda_{2}}\right)\right)\|w\|_{2}^{2}
$$

must hold for every $\delta \in\left[\delta_{(1)}, \delta^{(1)}\right]$ and $w(\cdot) \in \mathscr{D}_{1}$. Moreover, with the aid of Lemma 2, we see that there is a $\bar{\delta} \in\left(\delta_{(1)}, \delta^{(1)}\right)$ with the property that for every $\delta \in\left(\bar{\delta}, \delta^{(1)}\right]$ the expression $\sigma_{\lambda_{1}} l+k\left(1-\lambda_{2}^{-1}\right)$ is positive. Therefore, since $L^{2}(\mathscr{B})=$ $\mathscr{D}_{1} \oplus\left\{\alpha \phi_{1}(\cdot): \alpha \in \mathbb{R}\right\}$, we may conclude from Theorem 5 that

$$
\delta^{2} E_{\delta}(u ; \eta)>0
$$

holds for every $\eta(\cdot) \in \mathscr{A}_{0}^{1, p}(\mathscr{B})$ and $\delta \in(\bar{\delta}, \infty)$, and therefore, the extremal field $u(\cdot) \in \mathscr{A}^{1, p}(\mathscr{B})$ of $(21)$ is locally stable. For the particular choice of material parameters $k=a$ and $l=1$, this analysis yields a marked improvement on $\delta^{(1)}$ as a lower bound for local stability. For this case, we have

$$
\begin{aligned}
& \delta^{*}=(2 \ln 2)^{-1} \simeq 0.7213, \\
& \delta^{(1)}=\frac{2}{\pi} \simeq 0.6366, \\
& \delta_{(1)}=\frac{2}{3 \pi} \simeq 0.2122,
\end{aligned}
$$


and, by numerical analysis of the expression $\sigma_{\lambda_{1}} l+k\left(1-\lambda_{2}^{-1}\right)$ in (135), we find that $\bar{\delta} \simeq 0.35$. This means that for any $\delta>0.35$ the extremal field $u(\cdot) \in \mathscr{A}^{1, p}(\mathscr{B})$ that solves the Euler-Lagrange equation (21) is locally stable. Earlier, we had found this to be true for any $\delta \geq \delta^{(1)}$ and so an improvement has been accomplished. To completely answer the question of stability for the remaining "stability gap" $\left[\delta_{(1)}, \bar{\delta}\right]$, a more thorough study of (134) should be completed, but we do not attempt this here.

Acknowledgments. R.L.F. gratefully acknowledges the NSF under grant MSS-9024637 and Alliant Tech Systems Inc. for their partial support of this research. D.E.M. also wishes to acknowledge the NSF for their support through a National Science Foundation Graduate Research Fellowship (1993-1995). We thank R.C. Rogers for his helpful comments and discussions.

\section{REFERENCES}

[1] S. S. Antman, Monotonicity and invertibility conditions in one dimensional nonlinear elasticity. In: Nonlinear Elasticity, Proceedings of a Symposium Conducted by the Mathematics Research Center, University of Wisconsin-Madison, ed. by R. W. Dickey, Academic Press, 1973, pp. 57-92

[2] S. S. Antman, Ordinary differential equations of nonlinear elasticity II: Existence and regularity theory for conservative boundary value problems, Arch. Rational Mech. Anal. 61, 353-393 (1976)

[3] J. Carr, M. E. Gurtin, and M. Slemrod, Structured phase transitions on a finite interval, Arch. Rational Mech. Anal. 86, 317-351 (1984)

[4] R. Courant and D. Hilbert, Methods of Mathematical Physics, Vol. I, John Wiley and Sons, 1962

[5] B. Dacorogna, Direct Methods in the Calculus of Variations, Springer-Verlag, 1989

[6] J. E. Dunn and R. L. Fosdick, The morphology and stability of material phases, Arch. Rational Mech. Anal. 74, 1-99 (1980)

[7] I. Ekeland and R. Temam, Convex Analysis and Variational Problems. Studies in Mathematics and its Applications, Volume I, North-Holland Publishing Co., 1976

[8] J. L. Ericksen, Equilibrium of Bars, J. Elasticity 5, 191-201 (1975)

[9] R. L. Fosdick and D. E. Mason, Energy Minimizers in Nonlocal Continuum Mechanics (Work in progress)

[10] T. Lin and R. C. Rogers, Computation of Phase Transitions Using Nonlocal Regularization, ICAM Report, Virginia Polytechnic Institute, Department of Mathematics, 14 September 1993

[11] I. Stakgold, Boundary Value Problems of Mathematical Physics, Vol. I, The Macmillan Company, 1967

[12] J. D. van der Waals, The thermodynamic theory of capillarity under the hypothesis of a continuous variation of density. (In Dutch), Verhandel. Konink. Akad. Weten. Amsterdam (Sect. 1), 1, No. 8 (1893)

[13] J. W. Walter, On a Nonlocal Model of Fluid Phases, Ph.D. Thesis, University of Minnesota, Dept. of Aerospace Engineering and Mechanics, 1985

[14] R. L. Wheeden and A. Zygmund, Measure and Integral: An Introduction to Real Analysis, Marcel Dekker, Inc., 1977

[15] K. Yosida, Functional Analysis, Springer-Verlag, 1965 